\title{
ASYMPTOTIC DISTRIBUTION-FREE TESTS FOR SEMIPARAMETRIC REGRESSIONS WITH DEPENDENT DATA
}

\author{
By JuAn Carlos Escanciano ${ }^{1}$, JuAn CARlos PARdo-FernándeZ ${ }^{2}$ And \\ INGRID VAN KEILEGOM ${ }^{3}$
}

\section{Indiana University, Universidade de Vigo and KU Leuven}

\begin{abstract}
This article proposes a new general methodology for constructing nonparametric and semiparametric Asymptotically Distribution-Free (ADF) tests for semiparametric hypotheses in regression models for possibly dependent data coming from a strictly stationary process. Classical tests based on the difference between the estimated distributions of the restricted and unrestricted regression errors are not ADF. In this article, we introduce a novel transformation of this difference that leads to ADF tests with well-known critical values. The general methodology is illustrated with applications to testing for parametric models against nonparametric or semiparametric alternatives, and semiparametric constrained mean-variance models. Several Monte Carlo studies and an empirical application show that the finite sample performance of the proposed tests is satisfactory in moderate sample sizes.
\end{abstract}

1. Introduction. Let $Y_{t}$ be a response variable and $X_{t}$ a $d$-dimensional explanatory variable. Assume that the process $\left(X_{t}, Y_{t}\right), t=0, \pm 1, \pm 2, \ldots$, is strictly stationary and ergodic, and that $E\left(Y_{t}^{2}\right)<\infty$. Let $Y_{t}$ be related to $X_{t}$ through the heteroskedastic regression model

$$
Y_{t}=\mu\left(X_{t}\right)+\sigma\left(X_{t}\right) \varepsilon_{t},
$$

where $\mu(x)=E\left(Y_{t} \mid X_{t}=x\right)$ and $\sigma^{2}(x)=\operatorname{Var}\left(Y_{t} \mid X_{t}=x\right)$ are almost surely (a.s.) the conditional mean and the conditional variance of $Y_{t}$ given $X_{t}=x$, respectively, and $\varepsilon_{t}$ is an error term, which is independent of $X_{t}$. Let $\mu$ belong to a certain class of measurable functions, $\mathcal{M}$, that will be specified for each application. This class may consist of semiparametric or nonparametric specifications for $\mu$. In this article, we propose a general methodology for testing parametric or semiparametric hypotheses about the regression function $\mu$. That is, we are interested in testing

$$
H_{0}: \mu \in \mathcal{M}_{0} \quad \text { versus } \quad H_{1}: \mu \in \mathcal{M} \backslash \mathcal{M}_{0},
$$

Received July 2016; revised February 2017.

${ }^{1}$ Supported by the Spanish Plan Nacional de I+D+I, reference number ECO2014-55858-P.

${ }^{2}$ Supported by the Spanish Ministerio de Economía y Competitividad (Grant MTM2014-55966-P).

${ }^{3}$ Supported by the European Research Council (2016-2021, Horizon 2020 ERC Grant agreement No. 694409), and from IAP Research Network P7/06 of the Belgian State.

MSC2010 subject classifications. 62E20, 62G08, 62G20, 62H15.

Key words and phrases. Beta-mixing, error distribution, goodness-of-fit tests, local polynomial estimation, nonparametric regression. 
where

$$
\mathcal{M}_{0}=\left\{\mu: \mathbb{R}^{d} \rightarrow \mathbb{R} \text { such that } \mu(x)=g(\theta, \eta(x), x), \theta \in \Theta \subset \mathbb{R}^{r}, \eta \in \mathcal{H}\right\}
$$

is a subclass of $\mathcal{M}$ of parametric or semiparametric models defined in terms of a known function $g$, an unknown parameter $\theta \in \Theta \subset \mathbb{R}^{r}$ and a possibly infinitedimensional unknown parameter $\eta$ belonging to a class of functions $\mathcal{H}$.

This general formulation covers many testing problems in regression with a parametric/semiparametric null hypothesis versus a semiparametric/nonparametric alternative. In particular, if $g(\theta, \eta(x), x) \equiv g(\theta, x)$, then the problem reduces to testing for a parametric model for the regression function $\mu$, which is a classical problem in statistics. See, for instance, the review that [19] offers about this topic. Special cases of semiparametric null hypotheses include the partially linear model with $g(\theta, \eta(x), x)=\theta^{\prime} x_{1}+\eta\left(x_{2}\right), x^{\prime}=\left(x_{1}^{\prime}, x_{2}^{\prime}\right)$ (where $A^{\prime}$ denotes the transpose of $A)$, additive models with $g(\theta, \eta(x), x)=\eta_{1}\left(x_{1}\right)+\eta_{2}\left(x_{2}\right), \eta=\left(\eta_{1}, \eta_{2}\right)$, varying-coefficient models $g(\theta, \eta(x), x)=x_{2}^{\prime} \eta\left(x_{1}\right)$, and single-index models with $g(\theta, \eta(x), x)=\eta\left(\theta^{\prime} x\right)$, among many others. Our methodology can also be applied to semiparametric alternative hypotheses. For example, a researcher suspecting of nonlinear effects might be interested in testing the parametric linear model $g(\theta, \eta(x), x)=\theta_{1}^{\prime} x_{1}+\theta_{2}^{\prime} x_{2}, \theta^{\prime}=\left(\theta_{1}^{\prime}, \theta_{2}^{\prime}\right)$, against the partially linear model $g\left(\theta_{1}, \eta(x), x\right)=\theta_{1}^{\prime} x_{1}+\eta\left(x_{2}\right)$, or against the single-index model $g(\theta, \eta(x), x)=$ $\eta\left(\theta^{\prime} x\right)$. The proposed methodology can be equally applied to these different settings.

A new class of examples that we analyze below is that of constrained meanvariance models, where $\eta(x)=\sigma(x)$. The statistical properties of this class of semiparametric models have not been investigated in this generality before. Among other interesting specifications, the choice $g(\theta, \sigma(x), x)=\sigma(x) g_{1}(\theta, x)$ leads to a parametric model given by $g_{1}(\theta, x)$ for the nonparametric standardized first moment $\mu(x) / \sigma(x)$. When $Y_{t}$ is a stock return, $\mu(x) / \sigma(x)$ is called the Sharpe ratio, and its statistical analysis is of practical interest because it measures the stock return per unit of risk. Indeed, there is an extensive literature in financial time series on modeling and testing hypotheses on $\mu(x) / \sigma(x)$ (see, e.g., [29, 30]) but there are no statistical tests available for parametric specifications of $\mu(x) / \sigma(x)$ when $\mu(x)$ and $\sigma(x)$ are nonparametric.

Tests for parametric and some semiparametric hypotheses on the regression function have been investigated before in the literature, with a special focus on independent and identically distributed (i.i.d.) observations; see the above mentioned paper [19] for a recent comprehensive survey on the topic. The two main methodologies are based on comparisons of unrestricted (i.e., nonparametric) estimators of $\mu$ and restricted estimators of $\mu$ (see, e.g., [22]), or their corresponding cumulative processes (see, e.g., $[4,26,34,35]$ ). Test statistics in the first methodology have rates of convergence that depend on suitable bandwidth sequences and lead to tests which are unable to detect local alternatives converging at the parametric 
rate. Tests based on cumulative processes detect such local alternatives, although their asymptotic local power function is essentially flat (see [24]). Moreover, the "directions" where local power is nontrivial lack interpretation (see [11]). Directional tests that specify the direction of departure exist (see [34], Section 3 and [35]), and although they are easy to interpret, they are inconsistent against all but one fixed alternative.

A third methodology is based on the comparison of unrestricted and restricted estimators of the distributions of the standardized errors. This methodology has been used in several regression contexts in the recent literature, and it is widely applicable. For instance, [38] and [6] used this idea to develop goodness-of-fit tests for the parametric form of the regression function and the variance function, respectively. [7] developed goodness-of-fit tests for a multiplicative structure between the regression function and the scale function. Tests using this methodology have parametric rates of convergence, unlike tests based on direct comparisons of unrestricted and restricted estimates of $\mu$. However, their null limit distribution depends on the unknown density of the error distribution and, therefore, are not Asymptotically Distribution-Free (ADF). Smoothed bootstrap methods have been proposed to approximate the corresponding critical values. These bootstrap methods are computationally intensive and require the choice of additional smoothing parameters.

This article provides three main contributions within the context of the third methodology explained above. First, it proposes a novel transformation of the difference of error distributions that leads to ADF tests. Second, it widens the scope of applications of this third methodology to general semiparametric null and semiparametric/nonparametric alternative hypotheses. Compared to other existing ADF tests available in the literature for dependent data, such as Koul and Stute's [26] ADF test for parametric autoregressions, the proposed tests are ADF in a much more general setting that includes semiparametric null and semiparametric/nonparametric alternative hypotheses. Third, this article provides a global and local power analysis of tests based on the third methodology. We show that from the local point of view our tests are directional, with a nontrivial local power at the parametric rate that can be tailored to specific alternatives of interest, while from the global point of view our tests are consistent against all fixed global alternatives. Thus, the power properties of tests in the third methodology are fundamentally different from those of existing tests based on the first and second methodologies. Monte Carlo experiments show a satisfactory finite sample performance for the proposed tests in three different applications: parametric regression models, mean-variance constrained models and parametric linear regression models against semiparametric partially linear models.

The remainder of this article is organized as follows. In Section 2, we introduce the new methodology in a general context. In Section 3, we discuss practical implementation in several examples. In Section 4, we investigate the asymptotic power properties of the tests. In Section 5, we report some simulation studies and 
a real data example. Finally, we conclude in Section 6. All mathematical proofs are gathered in an Appendix and in the Supplementary Material [14].

\section{General methodology.}

2.1. Test statistics. This section introduces the general methodology. The discussion here is organized around a few "high-level" assumptions. More specific conditions can be given in specific applications; see Section 3 below for illustrations. Define the restricted model for the regression function as $\mu_{0}(x)=$ $g\left(\theta_{0}, \eta_{0}(x), x\right)$, where

$$
\left(\theta_{0}, \eta_{0}\right)=\arg \min _{\theta \in \Theta, \eta \in \mathcal{H}} E\left(\left(\mu\left(X_{t}\right)-g\left(\theta, \eta\left(X_{t}\right), X_{t}\right)\right)^{2}\right) .
$$

Henceforth, for the sake of identifiability, we assume that $\left(\theta_{0}, \eta_{0}\right)$ exists and is unique. Note that $H_{0}$ is equivalent to the fact that $\mu=\mu_{0}$. Then define the standardized errors

$$
\varepsilon_{t 0}=\frac{Y_{t}-\mu_{0}\left(X_{t}\right)}{\sigma\left(X_{t}\right)} \quad \text { and } \quad \varepsilon_{t}=\frac{Y_{t}-\mu\left(X_{t}\right)}{\sigma\left(X_{t}\right)},
$$

with cumulative distribution functions $F_{\varepsilon 0}(y)=P\left(\varepsilon_{t 0} \leq y\right)$ and $F_{\varepsilon}(y)=P\left(\varepsilon_{t} \leq\right.$ $y)$, respectively. We show in Theorem 1 below three equivalent formulations for $H_{0}$ which motivate the testing methodology introduced in this article. lent:

THEOREM 1. Assume that $E\left(\varepsilon_{t 0}^{2}\right)<\infty$. The following statements are equiva-

(i) $H_{0}$ is true;

(ii) $\varepsilon_{t 0}$ and $\varepsilon_{t}$ have the same distribution;

(iii) $D(y)=\int_{-\infty}^{y}\left(F_{\varepsilon 0}(s)-F_{\varepsilon}(s)\right) d s=0$ for all $y \in \mathbb{R}$.

The equivalence between statements (i) and (ii) has been used in recent literature to construct tests for specific examples of $H_{0}$ (see, e.g., [38] or [7]). In this article, we will exploit the equivalence between (i) and (iii), which, as we show below, will allow us to obtain ADF tests.

In practice, the variables $\varepsilon_{t}$ and $\varepsilon_{t 0}$ are not observable, so they need to be estimated. Assume that a sample $\left(X_{t}, Y_{t}\right), t=1, \ldots, T$, is available and construct the estimated residuals

$$
\widehat{\varepsilon}_{t 0}=\frac{Y_{t}-\widehat{\mu}_{0}\left(X_{t}\right)}{\widehat{\sigma}\left(X_{t}\right)} \quad \text { and } \quad \widehat{\varepsilon}_{t}=\frac{Y_{t}-\widehat{\mu}\left(X_{t}\right)}{\widehat{\sigma}\left(X_{t}\right)},
$$

for $t=1, \ldots, T$, where $\widehat{\mu}(x)$ is an estimator of $\mu(x)$ under model $\mathcal{M}, \widehat{\sigma}(x)$ is a consistent estimator of $\sigma(x)$ under model $\mathcal{M}$, and $\widehat{\mu}_{0}(x)$ is a suitable consistent estimator of $\mu_{0}(x)$ under model $\mathcal{M}_{0}$. For example, if $\mathcal{M}$ is fully nonparametric, then examples of estimators $\widehat{\mu}(x)$ and $\widehat{\sigma}(x)$ include kernel and sieve estimators. There 
are also general estimation methods available for semiparametric models that can be used to estimate $\left(\theta_{0}, \eta_{0}\right)$, and hence $\mu_{0}$. For example, we can use the sieve least squares estimators $(\widehat{\theta}, \widehat{\eta})=\arg \min _{\theta \in \Theta, \eta \in \mathcal{H}_{T}} T^{-1} \sum_{t=1}^{T}\left(Y_{t}-g\left(\theta, \eta\left(X_{t}\right), X_{t}\right)\right)^{2}$, where $\mathcal{H}_{T}$ is a sieve approximation of $\mathcal{H}$ (see, e.g., [2] and references therein). In other applications, alternative estimators for $\eta_{0}$, such as kernel estimators, can be used. This is the case for our constrained mean-variance example, where $\widehat{\eta}=\widehat{\sigma}$, and where $\theta_{0}$ can be estimated by the (two-step) semiparametric least squares estimator $\widehat{\theta}=\arg \min _{\theta \in \Theta} T^{-1} \sum_{t=1}^{T}\left(Y_{t}-g\left(\theta, \widehat{\sigma}\left(X_{t}\right), X_{t}\right)\right)^{2}$. Rather than focusing on a specific estimator or class of estimators, here we assume that consistent estimators for $\left(\mu, \sigma, \theta_{0}, \eta_{0}\right)$, say $(\widehat{\mu}, \widehat{\sigma}, \widehat{\theta}, \widehat{\eta})$, are available and satisfy certain conditions, and we refer to the detailed examples below for specific choices of estimators and verification of the conditions. The restricted estimator of $\mu_{0}(x)$ is then denoted by $\widehat{\mu}_{0}(x)=g(\widehat{\theta}, \widehat{\eta}(x), x)$.

The corresponding distribution functions $F_{\varepsilon 0}(y)$ and $F_{\varepsilon}(y)$ are estimated by

$$
\widehat{F}_{\varepsilon 0}(y)=\frac{1}{T \bar{w}} \sum_{t=1}^{T} w\left(X_{t}\right) I\left(\widehat{\varepsilon}_{t 0} \leq y\right) \quad \text { and } \quad \widehat{F}_{\varepsilon}(y)=\frac{1}{T \bar{w}} \sum_{t=1}^{T} w\left(X_{t}\right) I\left(\widehat{\varepsilon}_{t} \leq y\right),
$$

respectively, where $w$ is a positive weight function and $\bar{w}=T^{-1} \sum_{t=1}^{T} w\left(X_{t}\right)$. The weights are introduced as a technical device to allow for covariates with noncompact support. Note that when $w \equiv 1$, then the regular empirical distribution functions based on estimated residuals are obtained. Given the standardized difference of empirical distributions

$$
\widehat{R}(y)=\sqrt{T}\left(\widehat{F}_{\varepsilon 0}(y)-\widehat{F}_{\varepsilon}(y)\right),
$$

$-\infty<y<+\infty$, under suitable regularity conditions (see below) and the null hypothesis $H_{0}$, one can establish an asymptotic expansion for $\widehat{R}(y)$ as follows:

$$
\widehat{R}(y)=\frac{f_{\varepsilon}(y)}{E\left(w\left(X_{t}\right)\right)} T^{-1 / 2} \sum_{t=1}^{T} w\left(X_{t}\right) W_{t}+o_{P}(1),
$$

uniformly in $-\infty<y<\infty$, where $f_{\varepsilon}(y)$ is the density function corresponding to $F_{\varepsilon}(y)$, and $W_{t}$ is a zero-mean random variable, which will be defined later. We further assume that $0<\sigma_{W}^{2}<\infty$, where

$$
\sigma_{W}^{2}=\frac{E\left[w^{2}\left(X_{t}\right) W_{t}^{2}\right]}{\left(E\left(w\left(X_{t}\right)\right)\right)^{2}}
$$

The random variables $W_{t}$ and the regularity conditions needed for (3) to hold are of course specific to each application. For instance, [38] obtained this expansion to derive goodness-of-fit tests for parametric regression models, that is, for $g(\theta, \eta(x), x)=g(\theta, x)$, and used it to propose Kolmogorov-Smirnov (KS) and Cramér-von Mises (CM)-type statistics. Also [7] gave the analogous expansion and constructed tests for constrained mean-variance models when $g(\theta, \sigma(x), x)=$ 
$\theta \sigma(x)$ and $x$ is univariate. Tests based on the expansion of $\widehat{R}(y)$ have unknown null limiting distributions, as they depend on the density of the errors $f_{\varepsilon}(y)$ and other unknown quantities. Both [38] and [7] suggested to implement the tests with the assistance of a smoothed bootstrap procedure, which requires simulations and an additional bandwidth choice.

This article introduces a novel transformation of $\widehat{R}(y)$ that leads to ADF tests. Specifically, we consider the integrated process

$$
\widehat{C}(y)=\int_{-\infty}^{y} \widehat{R}(s) d s,
$$

$-\infty<y<\infty$, and show that the expansion

$$
\widehat{C}(y)=\frac{F_{\varepsilon}(y)}{E\left(w\left(X_{t}\right)\right)} T^{-1 / 2} \sum_{t=1}^{T} w\left(X_{t}\right) W_{t}+o_{P}(1),
$$

holds uniformly in $-\infty<y<\infty$ under the null hypothesis (see Theorem 2 below). This expansion allows to easily obtain the asymptotic null distribution of test statistics that are continuous functionals of $\widehat{C}(\cdot)$. In particular, taking into account that $\sup _{-\infty<y<\infty}\left|F_{\varepsilon}(y)\right|=1$ and $\int_{-\infty}^{\infty} F_{\varepsilon}^{2}(y) d F_{\varepsilon}(y)=1 / 3$, the expansion above allows us to obtain the asymptotic null distribution of the KS and CM-type statistics

$$
K S_{T}=\sup _{-\infty<y<\infty} \frac{1}{\widehat{\sigma}_{W}}|\widehat{C}(y)| \text { and } \quad C M_{T}=\frac{3}{\widehat{\sigma}_{W}^{2}} \int_{-\infty}^{\infty}(\widehat{C}(y))^{2} d \widehat{F}_{\varepsilon 0}(y),
$$

where $\widehat{\sigma}_{W}^{2}$ is a consistent estimator of $\sigma_{W}^{2}$ in (4), by essentially using the central limit theorem over the asymptotic expansion. We show in the next section that, under $H_{0}$,

$$
K S_{T} \rightarrow_{d}|Z| \text { and } C M_{T} \rightarrow_{d} Z^{2},
$$

where $Z \sim N(0,1)$. Therefore, the proposed tests are very easy to implement for two reasons. First, test statistics are straightforward to compute, since (5) has a closed-form expression as

$$
\widehat{C}(y)=\frac{1}{\sqrt{T} \bar{w}}\left(\sum_{s=1}^{T} w\left(X_{s}\right)\left\{\left(y-\widehat{\varepsilon}_{s}\right)_{+}-\left(y-\widehat{\varepsilon}_{s}\right)_{+}\right\}\right),
$$

where $a_{+}=\max \{a, 0\}$ and, therefore,

$$
\begin{aligned}
K S_{T} & =\max _{1 \leq t \leq T} \frac{1}{\widehat{\sigma}_{W}} \max \left\{\left|\widehat{C}\left(\widehat{\varepsilon}_{t}\right)\right|,\left|\widehat{C}\left(\widehat{\varepsilon}_{t 0}\right)\right|\right\}, \\
C M_{T} & =\frac{3}{\widehat{\sigma}_{W}^{2}} \frac{1}{T \bar{w}} \sum_{t=1}^{T} w\left(X_{t}\right)\left(\widehat{C}\left(\widehat{\varepsilon}_{t 0}\right)\right)^{2} .
\end{aligned}
$$


Second, critical values are readily available from (6). For instance, the CM test rejects $H_{0}$ at significance level $\alpha$ if $C M_{T}>\chi_{1,1-\alpha}^{2}$ where $C M_{T}$ is computed as in (8) and $\chi_{1,1-\alpha}^{2}$ is the $(1-\alpha)$-quantile of the chi-square distribution with one degree of freedom.

2.2. A general theory. We investigate now the limit distribution of the test statistics proposed in the previous section under a general set of regularity conditions. To that end, we introduce the following notation. Let $F_{X}(x)=P\left(X_{t} \leq x\right)$ and let $F(x, y)=P\left(X_{t} \leq x, Y_{t} \leq y\right)$ (which under Assumption G1 below do not depend on $t$ ). Lowercase letters will be used to denote the corresponding density functions. Define the $\beta$-mixing coefficients as (see, e.g., [9])

$$
\beta_{t}=\sup _{m \in \mathbb{Z}} \sup _{A \in \mathcal{F}_{t+m}^{\infty}} E\left|P\left(A \mid \mathcal{F}_{-\infty}^{m}\right)-P(A)\right|
$$

where $\mathcal{F}_{s}^{t}$ denotes the $\sigma$-algebra generated by the sequence $\left\{\left(X_{j}, Y_{j}\right), j=s, \ldots, t\right\}$ for $s \leq t$. Henceforth, $C$ is a generic constant that may change from expression to expression.

Assumption G1. The process $\left(X_{t}, Y_{t}\right), t=0, \pm 1, \pm 2, \ldots$, satisfies (1) and is strictly stationary and absolutely regular ( $\beta$-mixing), with mixing coefficients satisfying $\beta_{t}=O\left(t^{-b}\right)$, for some $b>2$.

ASSUMPTION G2. (i) The weight function $w$ has a compact support $R_{w}$ in $\mathbb{R}^{d}$ and satisfies $w(x)>0$ for all $x \in R_{w}$ and $\sup _{x \in R_{w}} w(x) \leq C$;

(ii) $\varepsilon_{t}$ is continuous with density $f_{\varepsilon}(y)$ satisfying $\sup _{y} f_{\varepsilon}(y)<\infty$ and $\sup _{y}\left|y f_{\varepsilon}(y)\right|<\infty$;

(iii) The errors of the regression model satisfy $E\left(\varepsilon_{t} \mid X_{t}, \mathcal{F}_{-\infty}^{t-1}\right)=E\left(\varepsilon_{t} \mid X_{t}\right)$ and $\operatorname{Var}\left(\varepsilon_{t} \mid X_{t}, \mathcal{F}_{-\infty}^{t-1}\right)=E\left(\varepsilon_{t}^{2} \mid X_{t}\right)$ a.s. Furthermore, $\varepsilon_{t}$ is independent of $X_{t}$, with mean zero and unit variance;

(iv) $\inf _{x \in R_{w}} \sigma(x)>0$.

Whenever there is no ambiguity, we use the same notation for any function of $X$ as for its version restricted to the compact set $R_{w}$. The class of smooth functions $C_{M}^{\tau}\left(R_{w}\right)$ is defined in the Appendix (see also p. 154 in [37]).

ASSUMPTION G3. The unrestricted estimators $\widehat{\mu}(x)$ and $\widehat{\sigma}(x)$ satisfy:

(i) $\sup _{x \in R_{w}}|\widehat{\mu}(x)-\mu(x)|=o_{P}\left(T^{-1 / 4}\right)$ and $\sup _{x \in R_{w}}|\widehat{\sigma}(x)-\sigma(x)|=$ $o_{P}\left(T^{-1 / 4}\right)$;

(ii) $P\left(\widehat{\mu}-\mu \in C_{M}^{\tau}\left(R_{w}\right)\right) \rightarrow 1$ as $T$ tends to infinity, where $\tau>d / 2$; 
(iii) For a measurable function $l(\cdot)$ satisfying $E\left(l\left(Y_{t}, X_{t}\right) \mid X_{t}, \mathcal{F}_{-\infty}^{t-1}\right)=0$ a.s. and $E\left(l^{2}\left(Y_{t}, X_{t}\right)\right)<\infty$, we have

$$
\int \frac{w(x)}{\sigma(x)}(\widehat{\mu}(x)-\mu(x)) d F_{X}(x)=T^{-1} \sum_{t=1}^{T} w\left(X_{t}\right) l\left(Y_{t}, X_{t}\right)+o_{P}\left(T^{-1 / 2}\right) .
$$

Assumption G4. Under $H_{0}$, the restricted estimator $\widehat{\mu}_{0}(x)=g(\widehat{\theta}, \widehat{\eta}(x), x)$ satisfies:

(i) $\sup _{x \in R_{w}}\left|\widehat{\mu}_{0}(x)-\mu_{0}(x)\right|=o_{P}\left(T^{-1 / 4}\right)$;

(ii) $P\left(\widehat{\mu}_{0}-\mu_{0} \in C_{M}^{\tau}\left(R_{w}\right)\right) \rightarrow 1$ as $T$ tends to infinity, where $\tau>d / 2$;

(iii) For a measurable function $l_{0}(\cdot)$ satisfying $E\left(l_{0}\left(Y_{t}, X_{t}\right) \mid X_{t}, \mathcal{F}_{-\infty}^{t-1}\right)=0$ a.s. and $E\left(l_{0}^{2}\left(Y_{t}, X_{t}\right)\right)<\infty$, we have

$$
\int \frac{w(x)}{\sigma(x)}\left(\widehat{\mu}_{0}(x)-\mu_{0}(x)\right) d F_{X}(x)=T^{-1} \sum_{t=1}^{T} w\left(X_{t}\right) l_{0}\left(Y_{t}, X_{t}\right)+o_{P}\left(T^{-1 / 2}\right) .
$$

Assumptions G1-G4 are standard in the literature. If $\widehat{\mu}(x), \widehat{\mu}_{0}(x)$ and $\widehat{\sigma}(x)$ are kernel estimators, there are well-known conditions for these assumptions to hold; see, for example, [20] for G3(i) and G4(i), and Lemma A.1 in [31] for G3(ii) and G4(ii). Note that [31] assume that the data are i.i.d., whereas here we have dependent data. However, the proof of their Lemma A.1 is based on [28], which is also valid for dependent data. G3(iii) and G4(iii) require a linear asymptotic representation for a linear functional of the estimators. For many examples of nonparametric/semiparametric models and estimators this representation has been already established. [32] (p. 1361) can be used to obtain the expression for $l\left(Y_{t}, X_{t}\right)$ and $l_{0}\left(Y_{t}, X_{t}\right)$ in a general setting. We verify these conditions for our leading examples below. In particular, as we will show later (see Lemma 4 in Section 3), if $\mathcal{M}$ is fully nonparametric and $\widehat{\mu}$ is a kernel-based estimator, then G3(iii) holds with $l\left(Y_{t}, X_{t}\right)=\left(Y_{t}-\mu\left(X_{t}\right)\right) / \sigma\left(X_{t}\right)=\varepsilon_{t}$.

Our next result establishes a uniform expansion of the process $\widehat{C}(y)$.

THEOREM 2. Assume G1-G4. Then, under the null hypothesis $H_{0}$ the following holds:

$$
\widehat{C}(y)=T^{1 / 2} \int_{-\infty}^{y}\left[\widehat{F}_{\varepsilon 0}(s)-\widehat{F}_{\varepsilon}(s)\right] d s=\frac{F_{\varepsilon}(y)}{E\left(w\left(X_{t}\right)\right)} T^{-1 / 2} \sum_{t=1}^{T} w\left(X_{t}\right) W_{t}+o_{P}(1),
$$

uniformly in $-\infty<y<\infty$, where $W_{t}=l_{0}\left(Y_{t}, X_{t}\right)-l\left(Y_{t}, X_{t}\right)$.

Theorem 2 does not follow directly from (3), since the mapping $R \rightarrow$ $\int_{-\infty}^{y} R(s) d s$ is not continuous in the space of uniformly bounded functions. Also note that the rate of convergence of $\widehat{C}$ is $T^{-1 / 2}$ and it does not depend on the 
dimension of $X_{t}$, in contrast to alternative nonparametric tests based on direct comparisons of $\widehat{\mu}(x)$ and $\widehat{\mu}_{0}(x)$.

We now assume that a consistent estimator exists for $\sigma_{W}^{2}=E\left(w^{2}\left(X_{t}\right) W_{t}^{2}\right) /$ $\left(E\left(w\left(X_{t}\right)\right)\right)^{2}$.

AsSUMPTION G5. $\quad 0<\sigma_{W}^{2}<\infty$, and there exists a weakly consistent estimator for $\sigma_{W}^{2}$, say $\widehat{\sigma}_{W}^{2}$.

A natural candidate to estimate $\sigma_{W}^{2}$ is

$$
\widehat{\sigma}_{W}^{2}=\frac{1}{\bar{w}^{2} T} \sum_{t=1}^{T} w^{2}\left(X_{t}\right) \widehat{W}_{t}^{2},
$$

where $\widehat{W}_{t}=\widehat{l}_{0}\left(Y_{t}, X_{t}\right)-\widehat{l}\left(Y_{t}, X_{t}\right)$ is a suitable consistent estimator of $W_{t}$; see the examples below.

The following corollary is a consequence of Theorem 2, the continuous mapping theorem, the central limit theorem for mixing sequences (see, for instance, Theorem 2.20 in [16]) and the consistency of the estimator $\widehat{\sigma}_{W}^{2}$.

COROLlary 3. Assume G1-G5. Then, under the null hypothesis $H_{0}$,

$$
K S_{T} \rightarrow_{d}|Z| \text { and } C M_{T} \rightarrow_{d} Z^{2},
$$

where $Z \sim N(0,1)$.

3. Practical implementation and examples. This section discusses issues of practical implementation. In particular, it provides detailed specific formulae for the estimators, influence functions, asymptotic variances $\sigma_{W}^{2}$ and their estimators for several examples: (1) parametric models against nonparametric alternatives; (2) parametric linear models against partially linear alternatives; (3) parametric linear models against single-index alternatives and (4) several constrained meanvariance models. This section also provides primitive regularity conditions under which our general high-level conditions hold for each of these examples. For each example, we will explain how to estimate $\mu$ and $\mu_{0}$.

The implementation of our tests entails computing unrestricted and restricted residuals $\left(\widehat{\varepsilon}_{t}\right.$ and $\widehat{\varepsilon}_{t 0}$, resp.), the weights $w\left(X_{t}\right)$ and the variance estimator $\widehat{\sigma}_{W}^{2}$. For the latter, we use (8), with an estimated $W_{t}$. In all the examples below, $W_{t}$ is a continuous function of $\varepsilon_{t}$. In our implementations, we suggest to replace $\varepsilon_{t}$ by $\widehat{\varepsilon}_{t}$ in $W_{t}$, but it is also possible to use $\widehat{\varepsilon}_{t 0}$ instead, since we only need a consistent estimator of $\sigma_{W}^{2}$ under the null. Unreported Monte Carlo simulations suggest that the choice of $\widehat{\varepsilon}_{t}$ or $\widehat{\varepsilon}_{t 0}$ for estimating $\sigma_{W}^{2}$ has little effect on the finite sample performance of the test statistics. 
Although they will not be needed in all examples, we will start by defining nonparametric estimators of $\mu(\cdot)$ and $\sigma(\cdot)$ to ease the presentation. To estimate nonparametrically $\mu(x)$, we use local polynomial estimators, that is, $\widehat{\mu}(x)=\widehat{\alpha}_{0}(x)$, where $\widehat{\alpha}_{0}(x)$ is the first component of the vector $\widehat{\alpha}(x)$, which is the solution of the local minimization problem

$$
\min _{\alpha} \sum_{t=1}^{T}\left\{Y_{t}-P_{t}(\alpha, x, p)\right\}^{2} K_{h}\left(X_{t}-x\right),
$$

where $P_{t}(\alpha, x, p)$ is a polynomial of order $p$ built up with all $0 \leq i \leq p$ products of factors of the form $X_{j t}-x_{j}, j=1, \ldots, d$, and $d$ is the dimension of $x$. The vector $\alpha$ consists of all coefficients of this polynomial. Here, for $u=\left(u_{1}, \ldots, u_{d}\right) \in \mathbb{R}^{d}$, $K(u)=\prod_{j=1}^{d} k\left(u_{j}\right)$ is a $d$-dimensional product kernel, $k$ is a univariate kernel function, $h=\left(h_{1}, \ldots, h_{d}\right)$ is a $d$-dimensional bandwidth vector converging to zero when $T$ tends to infinity, and $K_{h}(u)=\prod_{j=1}^{d} k\left(u_{j} / h_{j}\right) / h_{j}$. To estimate $\sigma(x)$, define

$$
\widehat{\sigma}^{2}(x)=\widehat{\gamma}_{0}(x)-\widehat{\alpha}_{0}^{2}(x),
$$

where $\widehat{\gamma}_{0}$ is defined in the same way as $\widehat{\alpha}_{0}$, but with $Y_{t}$ replaced by $Y_{t}^{2}$ in (9), $t=1, \ldots, T$. We note that such an estimator of the conditional variance is not guaranteed to be positive, and we refer to [15] or [27] for alternative positive estimators.

We need assumptions that guarantee that Assumption G3 holds for the local polynomial estimators.

Assumption N1. (i) All partial derivatives of $F_{X}$ up to order $2 d+1$ exist on the interior of $R_{w}$, they are uniformly continuous and $\inf _{x \in R_{w}} f_{X}(x)>0$;

(ii) All partial derivatives of $\mu$ and $\sigma$ up to order $p+2$ exist on the interior of $R_{w}$, they are uniformly continuous.

Assumption N2. (i) $E\left(\left|Y_{0}\right|^{s}\right)<\infty$ and $\sup _{x \in R_{X}} E\left(\left|Y_{0}\right|^{s} \mid X_{0}=x\right)<\infty$ for some $s>2+2 /(b-2)$, where $b$ is as in Assumption G1;

(ii) There exists some $j^{\prime}$ such that for all $j \geq j^{\prime}$,

$$
\sup _{x_{0}, x_{j} \in R_{X}} E\left(\left|Y_{0} Y_{j}\right|^{2} \mid X_{0}=x_{0}, X_{j}=x_{j}\right) f_{j}\left(x_{0}, x_{j}\right)<\infty,
$$

where $f_{j}\left(x_{0}, x_{j}\right)$ denotes the joint density of $\left(X_{0}, X_{j}\right)$.

ASSUMPTION N3. The function $F(x, y)$ is continuous in $(x, y)$, and twice continuously differentiable with respect to $x$ and $y$. Let $L(x, y)$ denote generically the derivatives $\frac{\partial}{\partial x} F(x, y), \frac{\partial}{\partial y} F(x, y), \frac{\partial^{2}}{\partial x^{2}} F(x, y), \frac{\partial^{2}}{\partial y^{2}} F(x, y)$ and $\frac{\partial^{2}}{\partial x \partial y} F(x, y)$. Then $L(x, y)$ is continuous in $(x, y)$ and satisfies $\sup _{x, y}\left|y^{2} L(x, y)\right|<\infty$. 
Assumption N4. (i) For all $j=1, \ldots, d: h_{j} / h_{1} \rightarrow C_{j}$, with $0<C_{j}<\infty$, and the bandwidth $h_{1}$ satisfies $(\log T)^{-1} T^{\eta} h_{1}^{d} \rightarrow \infty$ for $\eta=\frac{b-1-d-(1+b) /(s-1)}{b+3-d-(1+b) /(s-1)}$, where $d, b$ and $s$ are such that $d<\frac{(b-2)(s-2-2 /(b-2))+s-2}{s-1}$, and with $b$ and $s$ as defined in Assumptions G1 and N2, respectively, $T h_{1}^{2 d+\delta} \rightarrow \infty$ for some small $\delta>0, T h_{1}^{2 p+2} \rightarrow 0$ for odd $p$ and $T h_{1}^{2 p+4} \rightarrow 0$ for even $p$;

(ii) The kernel $k$ is a symmetric probability density function on $[-1,1], k$ is $d$ times continuously differentiable, and $k^{(j)}( \pm 1)=0$ for $j=0, \ldots, d-1$.

Assumptions N1-N4 are standard in the literature of nonparametric regression estimation. Assumption N2 and Assumption N4(i) are taken from [20], and they ensure suitable uniform rates of convergence of the kernel estimators of $\mu(\cdot)$ and $\sigma(\cdot)$.

LEMMA 4. Assume that G1-G2 and N1-N4 hold. Then Assumption G3 holds for the local polynomial estimator $\widehat{\mu}(x)$ with $l\left(Y_{t}, X_{t}\right)=\left(Y_{t}-\mu\left(X_{t}\right)\right) / \sigma\left(X_{t}\right)=$ $\varepsilon_{t}$.

The proof is straightforward and is therefore omitted. We proceed to describe semiparametric estimators and regularity conditions for each of the examples enumerated at the beginning of the section.

3.1. Parametric models against nonparametric alternatives. Consider the specification $g(\theta, \eta(x), x)=g(\theta, x)$. The testing problem is then a goodnessof-fit test for a parametric form of the regression function $\mu(\cdot)$. The unknown parameter $\theta_{0}$ can be estimated by the nonlinear least squares estimator $\widehat{\theta}=$ $\arg \min _{\theta \in \Theta} \frac{1}{T} \sum_{t=1}^{T}\left(Y_{t}-g\left(\theta, X_{t}\right)\right)^{2}$. The following assumption is needed to verify Assumption G4 in this example.

AsSUMPTION E1. $\theta_{0}$ belongs to the interior of a compact subset $\Theta$ of $\mathbb{R}^{r}$. The function $g(\cdot, x)$ is continuously differentiable with respect to $\theta$ in a neighborhood of $\theta_{0}$, say $\Theta_{0}$, for all $x \in \mathbb{R}^{d}$, with derivative $g_{\theta t}(\theta)=\partial g\left(\theta, X_{t}\right) / \partial \theta$ satisfying that $E\left(g_{\theta t}\left(\theta_{0}\right) g_{\theta t}^{\prime}\left(\theta_{0}\right)\right)$ is finite and nonsingular. Also, $l_{0}\left(Y_{t}, X_{t}\right)$ defined in (11) satisfies $E\left(l_{0}^{2}\left(Y_{t}, X_{t}\right)\right)<\infty$. Furthermore, for all $\theta \in \Theta_{0}, g(\theta, \cdot) \in C_{M}^{\tau}\left(R_{w}\right)$, for some $\tau>\max (d / 2,1)$.

LEMmA 5. Assume that G1-G2 and E1 hold. Then Assumption G4 holds for the parametric estimator $\widehat{\mu}_{0}(x)=g(\widehat{\theta}, x)$ with

$$
\begin{aligned}
l_{0}\left(Y_{t}, X_{t}\right)= & w^{-1}\left(X_{t}\right) E\left(w\left(X_{t}\right) \sigma^{-1}\left(X_{t}\right) g_{\theta t}^{\prime}\left(\theta_{0}\right)\right) \\
& \times\left(E\left(g_{\theta t}\left(\theta_{0}\right) g_{\theta t}^{\prime}\left(\theta_{0}\right)\right)\right)^{-1} g_{\theta t}\left(\theta_{0}\right) \sigma\left(X_{t}\right) \varepsilon_{t} .
\end{aligned}
$$


In the particular case of testing for a linear model $g(\theta, x)=\theta^{\prime} x$ and $g_{\theta t}\left(\theta_{0}\right)=$ $X_{t}$ and thus $l_{0}\left(Y_{t}, X_{t}\right)=w^{-1}\left(X_{t}\right) E\left(w\left(X_{t}\right) \sigma^{-1}\left(X_{t}\right) X_{t}^{\prime}\right)\left(E\left(X_{t} X_{t}^{\prime}\right)\right)^{-1} X_{t} \sigma\left(X_{t}\right) \varepsilon_{t}$. If the alternative is nonparametric, then $l\left(Y_{t}, X_{t}\right)=\varepsilon_{t}$. Hence,

$$
W_{t}=\varepsilon_{t}\left\{w^{-1}\left(X_{t}\right) E\left(w\left(X_{t}\right) \sigma^{-1}\left(X_{t}\right) X_{t}^{\prime}\right)\left(E\left(X_{t} X_{t}^{\prime}\right)\right)^{-1} X_{t} \sigma\left(X_{t}\right)-1\right\} .
$$

A consistent estimator for $\sigma_{W}^{2}$ is then obtained by replacing $\sigma\left(X_{t}\right)$ and $\varepsilon_{t}$ in the previous expression by $\widehat{\sigma}\left(X_{t}\right)$ and $\widehat{\varepsilon}_{t}$, respectively, and then using equation (8).

From a practical point of view, for the goodness-of-fit problem it is also recommended to apply some smoothing to the restricted estimator of $\mu$ as in [38]. That is, in the definition of $\varepsilon_{t 0}$ in $(2)$, replace $\widehat{\mu}_{0}\left(X_{t}\right)$ by $\widetilde{\mu_{0}}\left(X_{t}\right)$, where $\widetilde{\mu_{0}}\left(X_{t}\right)$ is obtained in the same way as the nonparametric estimator $\widehat{\mu}\left(X_{t}\right)$, but replacing the responses $Y_{t}$ by $\widehat{\mu}_{0}\left(X_{t}\right)$. Our asymptotic theory is still valid when this modification is applied to the estimated residuals.

3.2. Parametric linear model against partially linear model. Our methodology can be also used to test for parametric models against semiparametric alternatives, such as partially linear models. Consider testing for the linear model $Y_{t}=\theta_{1}^{\prime} X_{1 t}+\theta_{2} X_{2 t}+\sigma \varepsilon_{t}, \theta^{\prime}=\left(\theta_{1}^{\prime}, \theta_{2}\right)$, against the partially linear model $Y_{t}=$ $\beta^{\prime} X_{1 t}+\eta\left(X_{2 t}\right)+\sigma \varepsilon_{t}$, with $\operatorname{dim}\left(X_{1 t}\right)=d-1$ and $\operatorname{dim}\left(X_{2 t}\right)=1$. Identifiability requires that $X_{1 t}$ does not contain an intercept. For simplicity and in order to avoid multivariate estimation of the conditional variance function, in this example we assume a constant error conditional variance, $\sigma^{2}$. Also, throughout this and the next subsection we assume $w \equiv 1$. For this partially linear model, we take the estimators of $\beta$ and $\eta$ given in [33], that is, the unrestricted estimator of $\mu$ is $\widehat{\mu}(x)=\widehat{\beta}^{\prime} x_{1}+\widehat{\eta}\left(x_{2}\right)$, where

$$
\widehat{\beta}=S_{X, X}^{-1} S_{X, Y} \quad \text { and } \quad \widehat{\eta}\left(x_{2}\right)=\frac{\sum_{t=1}^{T} k_{h}\left(X_{2 t}-x_{2}\right)\left(Y_{t}-\widehat{\beta}^{\prime} X_{1 t}\right)}{\sum_{s=1}^{T} k_{h}\left(X_{2 s}-x_{2}\right)},
$$

where $k_{h}(u)=h^{-1} k(u / h)$ is the rescaled kernel, $h>0$ is the smoothing parameter, $S_{X, X}=T^{-1} \sum_{t=1}^{T}\left(X_{1 t}-\widehat{m}_{X_{1}}\left(X_{2 t}\right)\right)\left(X_{1 t}-\widehat{m}_{X_{1}}\left(X_{2 t}\right)\right)^{\prime}$ and $S_{X, Y}=$ $T^{-1} \sum_{t=1}^{T}\left(X_{1 t}-\widehat{m}_{X_{1}}\left(X_{2 t}\right)\right)\left(X_{1 t}-\widehat{m}_{Y}\left(X_{2 t}\right)\right)^{\prime}$, with

$$
\widehat{m}_{X_{1}}\left(x_{2}\right)=\frac{\sum_{t=1}^{T} k_{h}\left(X_{2 t}-x_{2}\right) X_{1 t}}{\sum_{s=1}^{T} k_{h}\left(X_{2 s}-x_{2}\right)} \quad \text { and } \quad \widehat{m}_{Y}\left(x_{2}\right)=\frac{\sum_{t=1}^{T} k_{h}\left(X_{2 t}-x_{2}\right) Y_{t}}{\sum_{s=1}^{T} k_{h}\left(X_{2 s}-x_{2}\right)} \text {. }
$$

Note that $\widehat{m}_{X_{1}}\left(x_{2}\right)$ and $\widehat{m}_{Y}\left(x_{2}\right)$ are kernel estimators of $m_{X_{1}}\left(x_{2}\right)=E\left(X_{1 t} \mid X_{2 t}=\right.$ $\left.x_{2}\right)$ and $m_{Y}\left(x_{2}\right)=E\left(Y_{t} \mid X_{2 t}=x_{2}\right)$, respectively.

Assumption E2. Assume that the conditions of the Theorem in [33] (p. 939) are satisfied.

LEMma 6. Assume that G1-G2 and E2 hold. Then Assumption G3 holds for the partially linear estimator $\widehat{\mu}(x)$ with $l\left(Y_{t}, X_{t}\right)=\sigma^{-1}\left\{Y_{t}-m_{Y}\left(X_{2 t}\right)-\beta^{\prime}\left[X_{1 t}-\right.\right.$ $\left.\left.m_{X_{1}}\left(X_{2 t}\right)\right]\right\}=\varepsilon_{t}$. 
Taking into account the influence function of the linear model given in the previous section and that we are working under constant error variance, we obtain that $W_{t}=\left(E\left(X_{t}^{\prime}\right)\left(E\left(X_{t} X_{t}^{\prime}\right)\right)^{-1} X_{t}-1\right) \varepsilon_{t}$. An estimator of $\sigma_{W}^{2}$ is then obtained by taking (8) with $\widehat{W}_{t}=\left(\left\{T^{-1} \sum_{t=1}^{T} X_{t}^{\prime}\right\}\left\{T^{-1} \sum_{t=1}^{T} X_{t} X_{t}^{\prime}\right\}^{-1} X_{t}-1\right) \widehat{\varepsilon}_{t}$, where $\widehat{\sigma}^{2}=T^{-1} \sum_{t=1}^{T}\left(Y_{t}-\widehat{\mu}\left(X_{t}\right)\right)^{2}$ and $\widehat{\varepsilon}_{t}=\widehat{\sigma}^{-1}\left\{Y_{t}-\widehat{m}_{Y}\left(X_{2 t}\right)-\widehat{\beta}^{\prime}\left[X_{1 t}-\widehat{m}_{X_{1}}\left(X_{2 t}\right)\right]\right\}$.

3.3. Parametric linear model against single-index model. Next, we consider testing for the linear model $Y_{t}=X_{t}^{\prime} \theta+\sigma \varepsilon_{t}$, against the single-index model $Y_{t}=\eta\left(X_{t}^{\prime} \beta\right)+\sigma \varepsilon_{t}$. A natural estimator of $\beta$ under the single-index model is the semiparametric least squares estimator

$$
\widehat{\beta}=\arg \min _{\beta \in B} \frac{1}{T} \sum_{t=1}^{T}\left(Y_{t}-\widehat{\eta}\left(X_{t}^{\prime} \beta \mid \beta\right)\right)^{2} I\left(X_{t} \in A\right),
$$

where $A$ is a subset of $\mathbb{R}^{d}, \widehat{\eta}(u \mid \beta)$ is a kernel estimator of $\eta(u \mid \beta)=E\left(Y_{t} \mid X_{t}^{\prime} \beta=\right.$ $u)$ :

$$
\widehat{\eta}(u \mid \beta)=\sum_{t=1}^{T} \frac{k_{h}\left(u-X_{t}^{\prime} \beta\right) Y_{t}}{\sum_{s=1}^{T} k_{h}\left(u-X_{s}^{\prime} \beta\right)},
$$

and $B$ is a compact subset of the unit sphere in $\mathbb{R}^{d}$ (for identifiability). The estimator of $\mu(x)=\eta\left(x^{\prime} \beta\right)$ is then given by $\widehat{\mu}(x)=\widehat{\eta}\left(x^{\prime} \widehat{\beta}\right)$.

Assumption E3. (i) $F_{X}$ is continuous, and $f_{X}$ is bounded away from 0 on $A^{\delta}=\left\{x \in \mathbb{R}^{d}:\|x-A\| \leq \delta\right\}$, where $A$ is an open convex subset of $\mathbb{R}^{d}$ and $\delta>0$.

(ii) $\eta$ has two bounded, continuous derivatives on $\left\{x^{\prime} \beta: x \in A^{\delta}\right\}$. Assume that $\int \sup _{\beta \in B} \eta\left(x^{\prime} \beta \mid \beta\right) d F_{X}(x)<\infty$.

(iii) $k$ is supported on $(-1,1)$ and is a symmetric probability density function with a bounded derivative. Moreover, $T h^{2} \rightarrow \infty$ and $T h^{4} \rightarrow 0$.

(iv) $E\left|\varepsilon_{t}\right|^{m}=M_{m}<\infty$ for all $m$.

Assumption E3 is standard in the literature; see [21]. The bounded integral assumption in E3(ii) enables us to interchange integrals with derivatives with respect to $\beta$ in our next result.

LEMma 7. Assume that G1-G2 and E3 hold. Then Assumption G3 holds for the single-index estimator $\widehat{\mu}(x)$ with $l\left(Y_{t}, X_{t}\right)=\sigma^{-1}\left\{Y_{t}-\eta\left(X_{t}^{\prime} \beta\right)\right\}=\varepsilon_{t}$.

3.4. Constrained mean-variance models. This section illustrates the general methodology with an application to constrained mean-variance models. In these models, $\eta(x)=\sigma(x)$. [7] studied the special case $g(\theta, \sigma(x), x)=\theta \sigma(x)$ and developed bootstrap-based tests for the corresponding hypothesis under the assumption that the covariate is one-dimensional. See also [5] for an alternative test with 
i.i.d. data. Our more general formulation here is motivated from applications in economics and finance.

The general null hypothesis $H_{0}$ also incorporates as a special case the hypothesis that $\mu\left(X_{t}\right) / \sigma\left(X_{t}\right)$ follows a specific parametric model. For instance, if the null hypothesis is of a multiplicative form $g(\theta, \sigma(x), x)=\sigma(x) g_{1}(\theta, x)$, then $\mu\left(X_{t}\right) / \sigma\left(X_{t}\right)$ will have the parametric structure specified in $g_{1}$. Examples are the linear model with $g_{1}(\theta, x)=\theta^{\prime} x$ in [36], and the exponential model in [8] and [1], with $g_{1}(\theta, x)=\exp \left(\theta^{\prime} x\right)$. These examples will be treated in detail in Sections 3.4.1 and 3.4.2. Hence, our null hypothesis encompasses tests for the correct specification of a parametric coefficient of variation. To the best of our knowledge, such tests are not available in the literature.

In the general case, the parameter $\theta_{0}$ can be estimated by the following weighted least-squares (LS) estimator:

$$
\widehat{\theta}=\arg \min _{\theta \in \Theta} \sum_{t=1}^{T} w\left(X_{t}\right)\left(Y_{t}-g\left(\theta, \widehat{\sigma}\left(X_{t}\right), X_{t}\right)\right)^{2},
$$

where $\widehat{\sigma}(\cdot)$ is defined in (10). See also [13] for a related estimator of $\theta_{0}$ in a more general context. We now define $\widehat{\mu}_{0}(x)=g(\widehat{\theta}, \widehat{\sigma}(x), x)$. The following assumption is needed to verify Assumption G4 in this general example.

ASSUMPTION E4. The function $g(\theta, u, x)$ is continuously differentiable with respect to the components of $\theta$ and $u$, with derivatives $g_{\theta t}(\theta)=\frac{\partial}{\partial \theta} g\left(\theta, \sigma\left(X_{t}\right), X_{t}\right)$ and $g_{u t}(\theta)=\left.\frac{\partial}{\partial u} g\left(\theta, u, X_{t}\right)\right|_{u=\sigma\left(X_{t}\right)}$ satisfying that $E\left(g_{\theta t}\left(\theta_{0}\right) g_{\theta t}^{\prime}\left(\theta_{0}\right)\right)$ is finite and nonsingular, and that $E\left(g_{u t}^{2}\left(\theta_{0}\right)\right)<\infty$. Assume $E\left(\varepsilon_{t}^{4}\right)<\infty$. Furthermore, for all $\theta \in \Theta_{0}, P\left(g(\theta, \widehat{\sigma}(\cdot), \cdot) \in C_{M}^{\tau}\left(R_{w}\right)\right) \rightarrow 1$, for some $\tau>\max (d / 2,1)$.

Define $u_{t}=\varepsilon_{t}-0.5 g_{u t}\left(\theta_{0}\right)\left(\varepsilon_{t}^{2}-1\right), \quad S\left(\theta_{0}\right)=E\left[w\left(X_{t}\right) g_{\theta t}\left(\theta_{0}\right) g_{\theta t}^{\prime}\left(\theta_{0}\right)\right] /$ $E\left(w\left(X_{t}\right)\right), s\left(X_{t}, \varepsilon_{t}\right)=S^{-1}\left(\theta_{0}\right) \sigma\left(X_{t}\right) g_{\theta t}\left(\theta_{0}\right) u_{t}$, and

$$
\varphi(\theta)=\frac{1}{E\left(w\left(X_{t}\right)\right)} E\left(\frac{w\left(X_{t}\right)}{\sigma\left(X_{t}\right)} g_{\theta t}(\theta)\right) .
$$

Lemma 8. Assume that G1-G2, N1-N4 and E4 hold. Then Assumption G4 holds for the estimator $\widehat{\mu}_{0}(x)=g(\widehat{\theta}, \widehat{\sigma}(x), x)$ with

$$
l_{0}\left(Y_{t}, X_{t}\right)=\varphi^{\prime}\left(\theta_{0}\right) s\left(X_{t}, \varepsilon_{t}\right)+0.5 g_{u t}\left(\theta_{0}\right)\left(\varepsilon_{t}^{2}-1\right) \text {. }
$$

The first term $s\left(X_{t}, \varepsilon_{t}\right)$ arises in the linear expansion because (12) satisfies

$$
\widehat{\theta}-\theta_{0}=\frac{1}{E\left(w\left(X_{t}\right)\right) T} \sum_{t=1}^{T} w\left(X_{t}\right) s\left(X_{t}, \varepsilon_{t}\right)+o_{P}\left(T^{-1 / 2}\right),
$$

as shown in [13]. The second term in (13) accounts for the effect of estimating $\sigma$ in $g\left(\theta, \sigma\left(X_{t}\right), X_{t}\right)$. Therefore, for this example G4 holds. As given in Lemma 4, 
G3(iii) holds for the unrestricted nonparametric estimator with $l\left(Y_{t}, X_{t}\right)=\varepsilon_{t}$, and we thus obtain

$$
W_{t}=\varphi^{\prime}\left(\theta_{0}\right) s\left(X_{t}, \varepsilon_{t}\right)+0.5 g_{u t}\left(\theta_{0}\right)\left(\varepsilon_{t}^{2}-1\right)-\varepsilon_{t}=A\left(X_{t}\right) u_{t},
$$

where $A\left(X_{t}\right)=\varphi^{\prime}\left(\theta_{0}\right) S^{-1}\left(\theta_{0}\right) \sigma\left(X_{t}\right) g_{\theta t}\left(\theta_{0}\right)-1$. The variable $W_{t}$ can be consistently estimated by $\widehat{W}_{t}=\widehat{A}_{t} \widehat{u}_{t}$, with $\widehat{A}_{t}=\widehat{\varphi}^{\prime}(\widehat{\theta}) \widehat{S}^{-1}(\widehat{\theta}) \widehat{\sigma}\left(X_{t}\right) \widehat{g}_{\theta t}(\widehat{\theta})-1$,

$$
\widehat{u}_{t}=\widehat{\varepsilon}_{t}-0.5 \widehat{g}_{u t}(\widehat{\theta})\left(\widehat{\varepsilon}_{t}^{2}-1\right), \quad \widehat{\varphi}(\widehat{\theta})=\frac{1}{\bar{w} T} \sum_{t=1}^{T} \frac{w\left(X_{t}\right)}{\widehat{\sigma}\left(X_{t}\right)} \widehat{g}_{\theta t}(\widehat{\theta}),
$$

$\widehat{S}(\widehat{\theta})$ is a consistent estimate for $S\left(\theta_{0}\right)$,

$$
\widehat{g}_{u t}(\widehat{\theta})=\left.\frac{\partial g\left(\widehat{\theta}, u, X_{t}\right)}{\partial u}\right|_{u=\widehat{\sigma}\left(X_{t}\right)} \quad \text { and } \quad \widehat{g}_{\theta t}(\widehat{\theta})=\frac{\partial g\left(\widehat{\theta}, \widehat{\sigma}\left(X_{t}\right), X_{t}\right)}{\partial \theta} .
$$

It is straightforward to prove the consistency of $\widehat{\sigma}_{W}^{2}$ in a given application, as the following examples illustrate.

3.4.1. Linear model with time-varying coefficient of variation. If $g\left(\theta_{0}, \sigma(x)\right.$, $x)=\sigma(x) \theta_{0}^{\prime} x$, we estimate $\theta_{0}$ by the LS estimator

$$
\widehat{\theta}=\left(\sum_{t=1}^{T} w\left(X_{t}\right) X_{t} X_{t}^{\prime} \widehat{\sigma}^{2}\left(X_{t}\right)\right)^{-1} \sum_{t=1}^{T} w\left(X_{t}\right) Y_{t} X_{t} \widehat{\sigma}\left(X_{t}\right) .
$$

A consistent estimator for $\sigma_{W}^{2}$ is then given by (8) with $\widehat{W}_{t}=\widehat{A}\left(X_{t}\right) \widehat{u}_{t}$, where $\widehat{u}_{t}=\widehat{\varepsilon}_{t}-0.5 \widehat{\theta}^{\prime} X_{t}\left(\widehat{\varepsilon}_{t}^{2}-1\right)$,

$$
\begin{aligned}
\widehat{S} & =\frac{1}{T} \sum_{t=1}^{T} w\left(X_{t}\right) \widehat{\sigma}^{2}\left(X_{t}\right) X_{t} X_{t}^{\prime} \quad \text { and } \\
\widehat{A}\left(X_{t}\right) & =\left[\frac{1}{T} \sum_{t=1}^{T} w\left(X_{t}\right) X_{t}^{\prime}\right] \widehat{S}^{-1} \widehat{\sigma}^{2}\left(X_{t}\right) X_{t}-1 .
\end{aligned}
$$

By the uniform consistency of $\widehat{\sigma}^{2}(\cdot)$ and $\widehat{\mu}(\cdot)$ on $R_{w}$, and the consistency of $\widehat{\theta}$ it follows that $\widehat{\sigma}_{W}^{2}=\sigma_{W}^{2}+o_{P}(1)$.

3.4.2. Nonlinear model with time-varying coefficient of variation. A nonlinear specification for $\mu\left(X_{t}\right) / \sigma\left(X_{t}\right)$ that has been entertained is $g_{1}\left(\theta_{0}, x\right)=\exp \left(\theta_{0}^{\prime} x\right)$; see, for example, [8]. In this case, the LS estimator in (12) leads to a consistent estimator for $\theta_{0}$ and $\sigma_{W}^{2}$ can be estimated by (8) with $\widehat{W}_{t}=\widehat{A}\left(X_{t}\right) \widehat{u}_{t}$, where $\widehat{u}_{t}=$ $\widehat{\varepsilon}_{t}-0.5 \exp \left(\widehat{\theta}^{\prime} X_{t}\right)\left(\widehat{\varepsilon}_{t}^{2}-1\right), \widehat{A}\left(X_{t}\right)=\widehat{\varphi}_{1} \widehat{S}^{-1} \widehat{\sigma}^{2}\left(X_{t}\right) X_{t} \exp \left(\widehat{\theta}^{\prime} X_{t}\right)-1$ :

$$
\begin{aligned}
\widehat{\varphi}_{1} & =\frac{1}{T} \sum_{t=1}^{T} w\left(X_{t}\right) X_{t}^{\prime} \exp \left(\widehat{\theta}^{\prime} X_{t}\right) \text { and } \\
\widehat{S} & =\frac{1}{T} \sum_{t=1}^{T} w\left(X_{t}\right) \widehat{\sigma}^{2}\left(X_{t}\right) X_{t} X_{t}^{\prime} \exp \left(2 \widehat{\theta}^{\prime} X_{t}\right) .
\end{aligned}
$$


4. Asymptotic power properties. This section investigates the asymptotic power of the proposed tests. We first analyze the power against the fixed alternatives:

$$
H_{1}: \mu(\cdot) \neq \mu_{0}(\cdot) \quad \text { with positive probability on } R_{w} .
$$

That is, under this alternative hypothesis $H_{1}, P\left(\mu\left(X_{t}\right) \neq \mu_{0}\left(X_{t}\right) \mid X_{t} \in R_{w}\right)>0$. We shall show that under certain conditions the proposed test statistics will diverge to infinity under $H_{1}$ as $T \rightarrow \infty$, thereby proving the consistency of the tests against these fixed alternatives. To that end, we show below in Proposition 9 that under the alternative hypothesis, uniformly in $-\infty<y<\infty$,

$$
T^{-1 / 2} \widehat{C}(y)=\int_{-\infty}^{y} R(s) d s+o_{P}(1) \equiv C(y)+o_{P}(1),
$$

where $R(s)=E\left(w\left(X_{t}\right)\left\{I\left(\varepsilon_{t 0} \leq s\right)-I\left(\varepsilon_{t} \leq s\right)\right\}\right) / E\left(w\left(X_{t}\right)\right)$. Therefore, by the continuous mapping theorem,

$$
T^{-1} C M_{T}=\frac{3}{\sigma_{W}^{2}} \int_{-\infty}^{\infty}(C(y))^{2} d F_{\varepsilon 0}(y)+o_{P}(1)
$$

and

$$
T^{-1 / 2} K S_{T}=\sup _{-\infty<y<\infty} \frac{1}{\sigma_{W}}|C(y)|+o_{P}(1) .
$$

Thus, given that both $C(\cdot)$ and $F_{\varepsilon 0}(\cdot)$ are continuous under our conditions, it follows that the test statistics will diverge to infinity as $T \rightarrow \infty$ if $C(y) \neq 0$ for some $y \in \mathbb{R}$. The next result shows that this is the case under $H_{1}$. The following condition simplifies some of the previous assumptions.

Assumption G6. It holds that:

(i) $E\left(\varepsilon_{t 0}^{2}\right)<\infty$;

(ii) $\sup _{x \in R_{w}}|\widehat{\mu}(x)-\mu(x)|=o_{P}(1) ; \sup _{x \in R_{w}}|\widehat{\sigma}(x)-\sigma(x)|=o_{P}(1)$ and $\sup _{x \in R_{w}}\left|\widehat{\mu}_{0}(x)-\mu_{0}(x)\right|=o_{P}(1)$.

Assumption G6 is standard in the literature. If $\widehat{\mu}(x), \widehat{\mu}_{0}(x)$ and $\widehat{\sigma}(x)$ are kernel estimators, there are well-known conditions for Assumption G6(ii) to hold; see, for example, [20] and Section 3 of the present article.

Proposition 9. Assume that G1, G2, G5 and G6 hold. Then our tests are consistent against $H_{1}$, that is, the power of our tests tends to 1 when $T$ tends to infinity.

This shows that our tests are omnibus tests, in the sense that for any fixed alternative in $H_{1}$, that is, any possible direction $\mu(x)-\mu_{0}(x)$, the power of our tests goes to one as the sample size goes to infinity. 
We consider now the limiting distribution under the local alternative

$$
H_{1 T}(a): \mu(x)=\mu_{0}(x)+\frac{\sigma(x) a(x)}{\sqrt{T}},
$$

where $a \neq 0$ is the (standardized) direction of departure, such that $E\left(\mid a\left(X_{t} \mid\right)<\right.$ $\infty$ and $0<E\left[a^{2}\left(X_{t}\right) W_{t}^{2}\right]<\infty$. The following standard assumption is needed to control the behavior of the estimator $\widehat{\mu}_{0}(x)$ under $H_{1 T}(a)$.

ASSUMPTION G4BIS. Under $H_{1 T}(a)$, the restricted estimator $\widehat{\mu}_{0}(x)=$ $g(\widehat{\theta}, \widehat{\eta}(x), x)$ satisfies:

(i) $\sup _{x \in R_{w}}\left|\widehat{\mu}_{0}(x)-\mu(x)\right|=o_{P}\left(T^{-1 / 4}\right)$;

(ii) $P\left(\widehat{\mu}_{0}-\mu_{0} \in C_{M}^{\tau}\left(R_{w}\right)\right) \rightarrow 1$ as $T$ tends to infinity, where $\tau>d / 2$;

(iii) For a measurable function $l_{0}(\cdot)$ satisfying $E\left(l_{0}\left(Y_{t}, X_{t}\right) \mid X_{t}, \mathcal{F}_{-\infty}^{t-1}\right)=0$ a.s. and $E\left(l_{0}^{2}\left(Y_{t}, X_{t}\right)\right)<\infty$, we have

$$
\begin{aligned}
\int \frac{w(x)}{\sigma(x)}\left(\widehat{\mu}_{0}(x)-\mu_{0}(x)\right) d F_{X}(x)= & T^{-1} \sum_{t=1}^{T} w\left(X_{t}\right) l_{0}\left(Y_{t}, X_{t}\right) \\
& -T^{-1 / 2} \int d(x) a(x) d F_{X}(x)+o_{P}\left(T^{-1 / 2}\right),
\end{aligned}
$$

where $d(\cdot)$ depends on the estimator of $\theta_{0}$ used.

Define

$$
\delta(a)=\frac{E\left[\left(w\left(X_{t}\right)+d\left(X_{t}\right)\right) a\left(X_{t}\right)\right]}{\left(E\left[w^{2}\left(X_{t}\right) W_{t}^{2}\right]\right)^{1 / 2}} .
$$

Then we have the following asymptotic result under the local alternative $H_{1 T}(a)$.

COROllary 10. Assume G1-G3, G4bis and G5. Then, under the local alternative $H_{1 T}(a)$,

$$
K S_{T} \rightarrow_{d}|Z+\delta(a)| \quad \text { and } \quad C M_{T} \rightarrow_{d}(Z+\delta(a))^{2},
$$

where $Z \sim N(0,1)$.

This result shows that our tests are able to detect local alternatives converging at the parametric rate. Our tests are directional from a local perspective, that is, the tests are not consistent against all local alternatives, as there are directions $a$ for which $\delta(a)=0$. This is a generic property of directional tests, including those in [34] (Section 3) and [35]. On the other hand, note that our tests are consistent against all fixed alternatives and all local alternatives that converge at rate $r_{T}$ with $1 / r_{T}=o(\sqrt{T})$. In particular, for all alternatives of order $s_{T}$, with $s_{T}$ being the rate of convergence of $\widehat{\mu}(\cdot)-\widehat{\mu}_{0}(\cdot)$, the test statistics diverge to infinity. This is 
an important advantage of our test with respect to tests that are based on the distance between $\widehat{\mu}(\cdot)$ and $\widehat{\mu}_{0}(\cdot)$ like, for example, in [22], or the directional tests of [34] (Section 3) and [35] that are inconsistent against a large class of fixed alternatives.

Thus, our tests have the advantages of omnibus tests against fixed global alternatives, and the simplicity of interpretation of directional tests at local alternatives converging at the parametric rate, with local power functions that can be explicitly computed and tailored to specific alternatives of interest. Therefore, from the power point of view our tests have fundamentally different properties from those of existing tests. The following example illustrates these points.

EXAmPle (Parametric models, cont.). Suppose we aim to test the linear model $\mu(x)=\theta x$ against all fixed alternatives $\mu(x) \neq \theta x$ with positive probability on $R_{w}$ (where $R_{w}$ is a large compact set satisfying our conditions above). Consider, for instance, the CM-type test. Proposition 9 shows that our CM-type test is consistent against all fixed alternatives under the conditions of Section 3.1, that is, under $\mu(x) \neq \theta x$ with positive probability on $R_{w}, P\left(C M_{T}>\chi_{1,1-\alpha}^{2}\right) \rightarrow 1$ as $T \rightarrow \infty$. Now, consider, for example, a quadratic local alternative $\mu(x)=\theta x+T^{-1 / 2} x^{2}$. From the arguments of Section 3.1, it is straightforward to verify that Assumption G4bis(iii) holds with

$$
d\left(X_{t}\right)=-E\left(w\left(X_{t}\right) \sigma^{-1}\left(X_{t}\right) X_{t}\right)\left(E\left(X_{t}^{2}\right)\right)^{-1} X_{t} \sigma\left(X_{t}\right) .
$$

We show now that there is a choice of $w$ that makes the drift function $\delta(a) \neq 0$ for the quadratic local alternative $a(x)=\sigma^{-1}(x) x^{2}$. As in [25] (p. 996), we can assume without loss of generality that $E\left[d\left(X_{t}\right) a\left(X_{t}\right)\right]=0$. Then choose $w_{0}(x)=a(x) I\left(x \in R_{w}\right)$, for a compact set $R_{w}$ in the support of $X_{t}$, so that the corresponding drift is

$$
\delta(a)=\frac{E\left[w_{0}\left(X_{t}\right) a\left(X_{t}\right)\right]}{\left(E\left[w_{0}^{2}\left(X_{t}\right) W_{t}^{2}\right]\right)^{1 / 2}} \neq 0 .
$$

Thus, the CM test based on $w_{0}$ guarantees a nontrivial local power against quadratic local alternatives, with an asymptotic local power function given by $P\left(\chi_{1}^{2}(\delta(a))>\chi_{1,1-\alpha}^{2}\right)$, where $\chi_{1}^{2}(\delta(a))$ is chi-square distribution with one degree of freedom and noncentrality parameter $\delta(a)$. More generally, for any alternative $a$ such that $E\left[d\left(X_{t}\right) a\left(X_{t}\right)\right]=0$ and with a nonzero positive part, we can choose $w(x)=a_{+}(x) I\left(x \in R_{w}\right)$ for a suitable $R_{w}$ so that the drift term $\delta(a) \neq 0$. It is in this precise sense that the test can be tailored to specific local alternatives of interest, while preserving the omnibus feature against global alternatives. 


\section{Numerical examples.}

5.1. Monte Carlo simulations. In this section, we will illustrate the finite sample performance of the asymptotic distribution-free tests based on the Kolmogorov-Smirnov $\left(K S_{T}\right)$ and Cramér-von Mises $\left(C M_{T}\right)$ statistics for the applications described in the previous section. In all cases, the rejection probabilities are based on 2000 simulated data sets. The estimation of the finite-dimensional parameter involved in the specification given in the null hypothesis is done by using the least squares estimator, as in (12), and the variance $\sigma_{W}^{2}$ is estimated as explained in (8). Nonparametric estimators of $\mu(\cdot)$ and $\sigma(\cdot)$ are obtained by local-linear estimation and Nadaraya-Watson estimation, respectively, with fixed bandwidths and least-squares cross-validation bandwidths (indicated as "c-v" in the tables). The nominal level is 0.05 in all cases. Some further numerical results and discussion can be found in the Supplementary Material [14].

5.1.1. Goodness-of-fit tests for parametric models for $\mu$. In this first set of simulations, we will deal with the goodness-of-fit problem of parametric models for the regression function $\mu(\cdot)$. The null hypothesis is $H_{0}: \mu(x)=\theta x$. We generate i.i.d. samples of sizes $T=100$ and $T=200$ from the model proposed in [38]:

$$
Y=\theta X+a(X)+0.20(1+X) \varepsilon,
$$

where the covariate $X$ has a uniform distribution on $[0,1]$ and the error $\varepsilon$ is standard normal. The parameter is fixed at $\theta=1$. The term $a(X)$ gives different possibilities:

(i) $a(x)=0$;

(ii) $a(x)=x^{2}$;

(iii) $a(x)=0.5 x \exp (x)$;

(iv) $a(x)=0.3 \sin (4 \pi x)$.

Model (i) is under the null hypothesis, whereas models (ii), (iii) and (iv) are under the alternative. In this case, since the covariate has compact support, the weight function is $w(x) \equiv 1$ and, therefore, the tests will be constructed on the basis of the regular empirical distributions.

Table 1 summarizes the obtained results for the proposed tests in comparison with the bootstrap-based tests in [38], which are denoted by $K S_{V K}$ and $C M_{V K}$. In order to make a fair comparison, we have adjusted the critical values of our tests to match the empirical levels of the bootstrap-based tests. The empirical powers are then size-corrected. To achieve that, instead of taking the critical values from the asymptotic distributions given in Corollary 3, we take them from the empirical distributions of the test statistics obtained from the 2000 simulations under the null hypothesis (model i). The critical value is chosen as the quantile that makes the level equal to the level of the bootstrap-based test and then that critical value 
TABLE 1

Observed rejection proportions in 2000 simulated data sets when the null hypothesis is $H_{0}: \mu(x)=\theta x$ with the tests based on $C M_{T}$ and $K S_{T}$ and those proposed in [38], indicated as $C M_{V K}$ and $K S_{V K}$. Model (i) is under the null; models (ii), (iii) and (iv) are under the alternative hypothesis. The significance level is 0.05 . The size of the asymptotic test is adjusted so as to equal the size of the bootstrap test

\begin{tabular}{|c|c|c|c|c|c|c|c|c|c|c|}
\hline \multirow[b]{3}{*}{ Model } & \multirow[b]{3}{*}{$\mathbf{h}$} & \multirow[b]{3}{*}{$\mathbf{T}:$} & \multicolumn{4}{|c|}{ Size-adjusted } & \multicolumn{4}{|c|}{ Van Keilegom et al. [38] } \\
\hline & & & \multicolumn{2}{|c|}{$K S_{T}$} & \multicolumn{2}{|c|}{$C M_{T}$} & \multicolumn{2}{|c|}{$K S_{V K}$} & \multicolumn{2}{|c|}{$C M_{V K}$} \\
\hline & & & 100 & 200 & 100 & 200 & 100 & 200 & 100 & 200 \\
\hline \multirow[t]{4}{*}{ (i) } & 0.15 & & 0.0455 & 0.0410 & 0.0410 & 0.0480 & 0.0455 & 0.0410 & 0.0410 & 0.0480 \\
\hline & 0.2 & & 0.0500 & 0.0445 & 0.0430 & 0.0455 & 0.0500 & 0.0445 & 0.0430 & 0.0455 \\
\hline & 0.25 & & 0.0520 & 0.0495 & 0.0460 & 0.0460 & 0.0520 & 0.0495 & 0.0460 & 0.0460 \\
\hline & $\mathrm{c}-\mathrm{v}$ & & 0.0600 & 0.0490 & 0.0450 & 0.0480 & 0.0600 & 0.0490 & 0.0450 & 0.0480 \\
\hline \multirow[t]{4}{*}{ (ii) } & 0.15 & & 0.8055 & 0.9850 & 0.8435 & 0.9935 & 0.7300 & 0.9715 & 0.8375 & 0.9905 \\
\hline & 0.2 & & 0.7915 & 0.9805 & 0.8330 & 0.9905 & 0.7350 & 0.9695 & 0.8210 & 0.9865 \\
\hline & 0.25 & & 0.7710 & 0.9730 & 0.8190 & 0.9855 & 0.7245 & 0.9660 & 0.7970 & 0.9805 \\
\hline & $\mathrm{c}-\mathrm{v}$ & & 0.8460 & 0.9885 & 0.8555 & 0.9945 & 0.7485 & 0.9725 & 0.8380 & 0.9905 \\
\hline \multirow[t]{4}{*}{ (iii) } & 0.15 & & 0.7330 & 0.9640 & 0.7925 & 0.9860 & 0.6855 & 0.9555 & 0.7910 & 0.9780 \\
\hline & 0.2 & & 0.7270 & 0.9585 & 0.7710 & 0.9765 & 0.6905 & 0.9480 & 0.7695 & 0.9720 \\
\hline & 0.25 & & 0.7040 & 0.9500 & 0.7585 & 0.9730 & 0.6775 & 0.9350 & 0.7410 & 0.9595 \\
\hline & $\mathrm{c}-\mathrm{v}$ & & 0.8015 & 0.9760 & 0.8125 & 0.9875 & 0.6950 & 0.9510 & 0.7800 & 0.9815 \\
\hline \multirow[t]{4}{*}{ (iv) } & 0.15 & & 0.6650 & 0.9115 & 0.3815 & 0.7795 & 0.8395 & 0.9965 & 0.8440 & 0.9980 \\
\hline & 0.2 & & 0.6675 & 0.9095 & 0.3880 & 0.7135 & 0.8135 & 0.9920 & 0.7930 & 0.9945 \\
\hline & 0.25 & & 0.6910 & 0.9275 & 0.4445 & 0.7535 & 0.7590 & 0.9810 & 0.7245 & 0.9835 \\
\hline & $\mathrm{c}-\mathrm{V}$ & & 0.7710 & 0.9440 & 0.4920 & 0.8670 & 0.8635 & 0.9965 & 0.8805 & 0.9990 \\
\hline
\end{tabular}

is employed to obtain the power in models (ii), (iii) and (iv). We observe that for models (ii) and (iii), the new tests yield better power than the bootstrap-based tests. On the other hand, model (iv) shows an advantage in favor of the bootstrap-based tests, especially in the case of the CM-type statistics. We must recall that the tests based on a bootstrap approximation require the choice of a second bandwidth and are more computationally demanding. In view of the results of this simulation, it seems that the proposed asymptotically distribution-free tests are reasonable competitors.

5.1.2. Parametric linear model against partially linear model. In this section, we will perform a simulation study to test for the validity of a linear model versus a partially linear model. The alternative hypothesis is therefore semiparametric. More precisely, the null hypothesis is

$$
H_{0}: \mu\left(x_{1}, x_{2}\right)=\theta_{1} x_{1}+\theta_{2} x_{2},
$$

and the alternative hypothesis is

$$
H_{1}: \mu\left(x_{1}, x_{2}\right)=\theta_{1} x_{1}+\eta\left(x_{2}\right),
$$


TABLE 2

Observed rejection proportions in 2000 simulated data sets testing the linear null hypothesis $H_{0}: \mu\left(x_{1}, x_{2}\right)=\theta_{1} x_{1}+\theta_{2} x_{2}$ versus the semiparametric alternative

$H_{1}: \mu\left(x_{1}, x_{2}\right)=\theta_{1} x_{1}+\eta\left(x_{2}\right)$. Model (i) is under the null hypothesis. Models (ii) and (iii) are under the alternative hypothesis. The significance level is 0.05

\begin{tabular}{|c|c|c|c|c|c|c|}
\hline \multirow[b]{2}{*}{ Model } & \multirow[b]{2}{*}{ h } & \multirow[b]{2}{*}{$\mathbf{T}$} & \multicolumn{2}{|c|}{$K S_{T}$} & \multicolumn{2}{|c|}{$C M_{T}$} \\
\hline & & & 200 & 500 & 200 & 500 \\
\hline \multirow[t]{4}{*}{ (i) } & 0.15 & & 0.0480 & 0.0560 & 0.0590 & 0.0550 \\
\hline & 0.20 & & 0.0480 & 0.0510 & 0.0500 & 0.0515 \\
\hline & 0.25 & & 0.0430 & 0.0490 & 0.0460 & 0.0485 \\
\hline & $\mathrm{c}-\mathrm{v}$ & & 0.0405 & 0.0460 & 0.0505 & 0.0530 \\
\hline \multirow[t]{4}{*}{ (ii) } & 0.15 & & 0.9680 & 1.000 & 0.8625 & 0.9985 \\
\hline & 0.20 & & 0.9595 & 1.000 & 0.8615 & 0.9975 \\
\hline & 0.25 & & 0.9555 & 1.000 & 0.8605 & 0.9975 \\
\hline & $\mathrm{c}-\mathrm{v}$ & & 0.9605 & 1.000 & 0.8670 & 0.9985 \\
\hline \multirow[t]{4}{*}{ (iii) } & 0.15 & & 0.9530 & 1.000 & 0.9185 & 0.9995 \\
\hline & 0.20 & & 0.9480 & 1.000 & 0.9200 & 0.9995 \\
\hline & 0.25 & & 0.9425 & 0.9995 & 0.9220 & 0.9995 \\
\hline & $\mathrm{c}-\mathrm{v}$ & & 0.9380 & 0.9995 & 0.9125 & 0.9995 \\
\hline
\end{tabular}

where $\eta$ is a smooth nonlinear function. We consider the following three specifications of $\mu$ :

(i) $\mu\left(x_{1}, x_{2}\right)=x_{1}+x_{2}$;

(ii) $\mu\left(x_{1}, x_{2}\right)=x_{1}+0.2 \sin \left(2 \pi x_{2}\right)$;

(iii) $\mu\left(x_{1}, x_{2}\right)=x_{1}+0.2 \exp \left(x_{2}\right)$.

Model (i) satisfies the null hypothesis, whereas models (ii) and (iii) are under the alternative hypothesis. In the simulations, the bidimensional covariate $\left(X_{1 t}, X_{2 t}\right)$ is drawn from two independent uniform distributions on $[0,1]$. We work under homoscedasticity with $\sigma=0.25$ and the error follows a standard normal distribution. Since the supports of the covariates are bounded, we take $w \equiv 1$. For the estimation of the partially linear model and the required quantities to construct the test statistics we follow the steps described in Section 3.2. Table 2 collects the results obtained from data sets with sample sizes $T=200$ and 500 .

Under model (i), a good approximation of the level is observed for both sample sizes. In terms of power-models (ii) and (iii) — the behavior is as expected: larger sample sizes produce larger power, with a small advantage of $K S_{T}$ over $C M_{T}$. Finally, it is worth noting that the choice of the smoothing parameter does not have relevant impact on the obtained results. The choice based on cross-validation behaves satisfactorily. 
5.1.3. Constrained mean-variance models. In this third set of simulations, we will investigate the practical performance of the proposed methodology to test for relationships between $\mu(\cdot)$ and $\sigma(\cdot)$. In this case, we consider data from pairs $\left(X_{t}, Y_{t}\right)$ with a dependence structure given by $X_{t}=Y_{t-1}, t \in \mathbb{Z}$. More precisely, sequences of sizes $T=200$ and $T=500$ are generated from the following data generating processes:

(i) $Y_{t}=0.1 X_{t}\left(1+0.2 X_{t}^{2}\right)^{1 / 2}+\left(1+0.2 X_{t}^{2}\right)^{1 / 2} \varepsilon_{t}$;

(ii) $Y_{t}=\exp \left(-0.1 X_{t}^{2}\right)\left(1+0.2 X_{t}^{2}\right)^{1 / 2}+\left(1+0.2 X_{t}^{2}\right)^{1 / 2} \varepsilon_{t}$;

(iii) $Y_{t}=0.2+\left(1+0.2 X_{t}^{2}\right)^{1 / 2} \varepsilon_{t}$;

(iv) $Y_{t}=\exp \left(-0.1 X_{t}^{4}\right)\left(1+0.2 X_{t}^{2}\right)^{1 / 2}+\left(1+0.2 X_{t}^{2}\right)^{1 / 2} \varepsilon_{t}$.

In this case, since the covariate has no compact support, the nonparametric estimation of the conditional mean and variance function are performed on the [5\%, 95\%] range of the covariate by conveniently adapting the weight function $w$. The values of the bandwidths are also adapted to the covariates.

Two null hypotheses will be tested: (a) $H_{0}: \mu(x)=\theta x \sigma(x)$, for which model (i) is under the null; and (b) $H_{0}: \mu(x)=\exp (-\theta x) \sigma(x)$, for which model (ii) is under the null. These specifications provide parametric models for the Sharpe ratio $\mu\left(X_{t}\right) / \sigma\left(X_{t}\right)$.

Table 3 displays the results. In the case of the null hypothesis (a) (left part of the table), the approximation of the level in model (i) is good and the behavior in terms of power is very satisfactory. The results for model (iii) show that $K S_{T}$ and $C M_{T}$ produce similar rejection probabilities. On the other hand, the right part of the table shows the results for the null hypothesis (b). In this case, the results for model (ii) show that although the level is overestimated for $T=200$, the approximation is reasonably good for $T=500$, especially for fixed values of the bandwidth. The cross-validation bandwidths still lead to an overestimation of the level. The power is excellent under both models (i) and (iii). Finally, although model (iv) is very close to the null hypothesis (b), we can see that both tests reach nontrivial power.

5.2. Real data example. As an illustration of our methodology, we will test parametric and semiparametric restrictions for the coefficient of variation or Sharpe ratio in a real data set. We consider the monthly continuously compounded return of the Center for Research on Security Prices (CRSP) value-weighted index (including dividends) during the period 1926-2008. The response variable $Y_{t}$ is the excess return constructed by subtracting to the CRSP index the monthly return on the 30-day Treasury Bill. The covariate is the one-month lagged value of the response, that is, $X_{t}=Y_{t-1}$. A detailed explanation of this data set can be found in [13], where estimation of a semiparametric index model was considered. In this application, we wish to test for several parametric and semiparametric specifications for the Sharpe ratio $\mu(x) / \sigma(x)$ in this data set. We consider three specifications: (i) linear $\mu(x) / \sigma(x)=\theta x$; (ii) exponential $\mu(x) / \sigma(x)=\exp \left(\theta^{\prime} x\right)$ and (iii) proportional to standard deviation $\mu(x) / \sigma(x)=\theta \sigma(x)$. The linear specification (i) 
TABLE 3

Observed rejection proportions in 2000 simulated data sets when the null hypothesis is (a) $H_{0}: \mu(x)=\theta x \sigma(x)$ [left panel, model (i) is under the null], or (b) $H_{0}: \mu(x)=\exp (-\theta x) \sigma(x)$ [right panel, model (ii) is under the null]. The significance level is 0.05 , and the sample size is $T=200$ and $T=500$

\begin{tabular}{|c|c|c|c|c|c|c|c|c|c|c|}
\hline \multirow[b]{3}{*}{ Model } & \multirow[b]{3}{*}{$\mathbf{h}$} & \multirow[b]{3}{*}{$\mathbf{T}:$} & \multicolumn{4}{|c|}{ (a) $H_{0}: \mu(x)=\theta x \sigma(x)$} & \multicolumn{4}{|c|}{ (b) $H_{0}: \mu(x)=\exp (-\theta x) \sigma(x)$} \\
\hline & & & \multicolumn{2}{|c|}{$K S_{T}$} & \multicolumn{2}{|c|}{$C M_{T}$} & \multicolumn{2}{|c|}{$K S_{V K}$} & \multicolumn{2}{|c|}{$C M_{V K}$} \\
\hline & & & 200 & 500 & 200 & 500 & 200 & 500 & 200 & 500 \\
\hline \multirow[t]{4}{*}{ (i) } & 0.50 & & 0.0575 & 0.0535 & 0.0565 & 0.0535 & 1.000 & 1.000 & 1.000 & 1.000 \\
\hline & 0.75 & & 0.0520 & 0.0515 & 0.0510 & 0.0515 & 1.000 & 1.000 & 1.000 & 1.000 \\
\hline & 1.00 & & 0.0500 & 0.0500 & 0.0490 & 0.0500 & 1.000 & 1.000 & 1.000 & 1.000 \\
\hline & $\mathrm{c}-\mathrm{v}$ & & 0.0565 & 0.0475 & 0.0545 & 0.0480 & 1.000 & 1.000 & 1.000 & 1.000 \\
\hline \multirow[t]{4}{*}{ (ii) } & 0.50 & & 1.000 & 1.000 & 1.000 & 1.000 & 0.0825 & 0.0535 & 0.0795 & 0.0540 \\
\hline & 0.75 & & 1.000 & 1.000 & 1.000 & 1.000 & 0.0680 & 0.0540 & 0.0715 & 0.0550 \\
\hline & 1.00 & & 1.000 & 1.000 & 1.000 & 1.000 & 0.0640 & 0.0590 & 0.0680 & 0.0600 \\
\hline & $\mathrm{c}-\mathrm{v}$ & & 1.000 & 1.000 & 1.000 & 1.000 & 0.0725 & 0.0710 & 0.0700 & 0.0715 \\
\hline \multirow[t]{4}{*}{ (iii) } & 0.50 & & 0.7335 & 0.9810 & 0.6735 & 0.9740 & 1.000 & 1.000 & 1.000 & 1.000 \\
\hline & 0.75 & & 0.7210 & 0.9810 & 0.6840 & 0.9780 & 1.000 & 1.000 & 1.000 & 1.000 \\
\hline & 1.00 & & 0.7125 & 0.9805 & 0.6825 & 0.9790 & 1.000 & 1.000 & 1.000 & 1.000 \\
\hline & $\mathrm{c}-\mathrm{v}$ & & 0.7170 & 0.9830 & 0.6755 & 0.9780 & 1.000 & 1.000 & 1.000 & 1.000 \\
\hline \multirow[t]{4}{*}{ (iv) } & 0.50 & & 1.000 & 1.000 & 1.000 & 1.000 & 0.2140 & 0.2900 & 0.1875 & 0.2595 \\
\hline & 0.75 & & 1.000 & 1.000 & 1.000 & 1.000 & 0.1715 & 0.2510 & 0.1580 & 0.2390 \\
\hline & 1.00 & & 1.000 & 1.000 & 1.000 & 1.000 & 0.1485 & 0.2255 & 0.1410 & 0.2175 \\
\hline & $\mathrm{c}-\mathrm{v}$ & & 1.000 & 1.000 & 1.000 & 1.000 & 0.1850 & 0.2655 & 0.1600 & 0.2420 \\
\hline
\end{tabular}

is very common in applied work; see, for example, [36]. The exponential model (ii) was considered in [8] and [1]. The proportional model (iii) is the most popular model, and it was introduced in the seminal work of [29,30]. Although there exists an extensive literature using and estimating these models (see, e.g., [17, 18, 23]), there are no nonparametric tests available to evaluate their goodness-of-fit.

We now apply our nonparametric tests to this data set. The sample size is 995 and the support of the covariate ranges between -0.31 and 0.67 , approximately. The tests are implemented on the [5\%,95\%] range of the covariate with a locallinear estimator for $\mu(\cdot)$ and a Nadaraya-Watson estimator for $\sigma(\cdot)$, with a common bandwidth obtained by cross-validation. The cross-validation bandwidth was $h_{c v}=0.048$. In order to check whether the choice of the bandwidth has an effect on the results, we have also performed the tests with bandwidths of the form $c h_{c v}$, where $c=0.5,1,1.5,2$. The results are collected in Table 4 . The reported $p$-values suggest that the linear and exponential specifications are rejected at $1 \%$ nominal level for all values of the bandwidth. In contrast, the tests fail to reject [29, 30]'s proportional model for all values of the bandwidth parameter. Thus, our empirical 
TABLE 4

Real data application: p-values of the tests based on $K S_{T}$ and $C M_{T}$ to test the specifications given in $H_{0}$. The smoothing parameters are of the form $c h_{c v}$, where $h_{c v}$ is the cross-validation bandwidth

\begin{tabular}{|c|c|c|c|c|c|c|}
\hline $\boldsymbol{H}_{\mathbf{0}}$ & Test statistic & c : & 0.5 & 1.0 & 1.5 & 2.0 \\
\hline \multirow[t]{2}{*}{$\mu(x) / \sigma(x)=\theta x$} & $K S_{T}$ & & 0.0010 & 0.0033 & 0.0063 & 0.0100 \\
\hline & $C M_{T}$ & & 0.0010 & 0.0029 & 0.0056 & 0.0086 \\
\hline \multirow[t]{2}{*}{$\mu(x) / \sigma(x)=\exp (-\theta x)$} & $K S_{T}$ & & 0.0000 & 0.0000 & 0.0000 & 0.0000 \\
\hline & $C M_{T}$ & & 0.0000 & 0.0000 & 0.0000 & 0.0000 \\
\hline \multirow[t]{2}{*}{$\mu(x) / \sigma(x)=\theta \sigma(x)$} & $K S_{T}$ & & 0.1999 & 0.4063 & 0.5922 & 0.7751 \\
\hline & $C M_{T}$ & & 0.2811 & 0.4971 & 0.6706 & 0.8162 \\
\hline
\end{tabular}

results confirm the suitability of the widely used proportional model in applied work.

6. Conclusions. In this article, we have proposed a general and simpleto-implement methodology for testing parametric or semiparametric hypotheses against semiparametric or nonparametric alternatives in regression models with possibly dependent data. The tests are based on the cumulative difference of the standardized error distributions under the null and alternative hypotheses, respectively. The asymptotic null distributions of the tests are known functionals of a standard normal random variable, for which critical values are readily available. The tests are consistent against fixed alternatives and are able to detect local alternatives converging to the null at the parametric rate. Some Monte Carlo experiments have shown a satisfactory finite sample performance for the tests in three different applications.

We now point out several topics for future research. We have not discussed the choice of the bandwidth parameters in our testing problem. Although there exists an extensive literature on bandwidth choice for estimation, there is no general theory available for testing purposes (see, e.g., the discussion in [19]). One possible approach in our context is to choose the bandwidth that maximizes the test statistic subject to convergence constraints on the bandwidth. This procedure is likely to be more stable for our methodology than for alternative nonparametric tests based on smoothers, since the rates of convergence of our tests do not depend on those of the bandwidth under standard rate conditions on bandwidth parameters. To apply these ideas, we would need to establish the expansion of Theorem 2 uniformly in the bandwidth parameters in a suitable range that converges to zero. This uniform expansion is feasible given existing results; see, for example, [10] and [12]. In any case, as shown in our simulations the impact of the smoothing parameter on the results seems rather mild, and the cross-validation bandwidth yielded satisfactory results.

The main contribution of this article is the use of the integrated empirical process of nonparametric residuals to obtain ADF tests for the regression function. 
Nevertheless, this idea goes beyond inference on the regression function, and it can be used more generally for inferences where suitable tests statistics are functionals of the error distribution. For example, using a similar idea to the one used in this article one can develop ADF nonparametric goodness-of-fit tests for the error distribution in a model with nonparametric conditional mean and variance. This application follows straightforwardly from our results and is of independent interest.

Another extension would be to semiparametric hypotheses on the conditional variance, as in [6]. Our transformation does not lead to asymptotic distribution-free tests in that context, but alternative transformations may exist.

\section{APPENDIX: PROOFS}

This Appendix contains the proofs of Theorems 1 and 2. The proofs of Lemmas 5-8, Proposition 9 and Corollary 10 can be found in the Supplementary Material [14].

Proof of TheOrem 1. The implications (i) $\Rightarrow$ (ii) $\Rightarrow$ (iii) are obvious. To prove (iii) $\Rightarrow$ (ii), it suffices to take the derivative of $D(y)$. Finally, let us prove (ii) $\Rightarrow$ (i). If $\varepsilon$ and $\varepsilon_{0}$ have the same distribution, then it also holds that $E(\varepsilon)=E\left(\varepsilon_{0}\right)$ and $\operatorname{Var}(\varepsilon)=\operatorname{Var}\left(\varepsilon_{0}\right)$. It is easy to see that $E\left(\varepsilon_{0}\right)=E(\varepsilon)+$ $E\left(\left(\mu\left(X_{t}\right)-\mu_{0}\left(X_{t}\right)\right) / \sigma\left(X_{t}\right)\right)$, and hence $E\left(\left(\mu\left(X_{t}\right)-\mu_{0}\left(X_{t}\right)\right) / \sigma\left(X_{t}\right)\right)=0$. On the other hand, we also have that $\operatorname{Var}\left(\varepsilon_{0}\right)=\operatorname{Var}(\varepsilon)+\operatorname{Var}\left[\left(\mu\left(X_{t}\right)-\mu_{0}\left(X_{t}\right)\right) / \sigma\left(X_{t}\right)\right]$, and hence $\operatorname{Var}\left[\left(\mu\left(X_{t}\right)-\mu_{0}\left(X_{t}\right)\right) / \sigma\left(X_{t}\right)\right]=0$. We can now conclude that $\left(\mu\left(X_{t}\right)-\right.$ $\left.\mu_{0}\left(X_{t}\right)\right) / \sigma\left(X_{t}\right)=0$ a.s., or, equivalently, $\mu\left(X_{t}\right)=\mu_{0}\left(X_{t}\right)$ a.s.

Proof of TheOREM 2. First, consider

$$
\begin{aligned}
& \frac{1}{T \bar{w}} \sum_{t=1}^{T} w\left(X_{t}\right) \int_{-\infty}^{y}\left\{I\left(\widehat{\varepsilon}_{t} \leq s\right)-I\left(\varepsilon_{t} \leq s\right)\right\} d s \\
& =\frac{1}{T \bar{w}} \sum_{t=1}^{T} w\left(X_{t}\right)\left\{I\left(\widehat{\varepsilon}_{t} \leq y\right)\left(y-\widehat{\varepsilon}_{t}\right)-I\left(\varepsilon_{t} \leq y\right)\left(y-\varepsilon_{t}\right)\right\} \\
& =\frac{1}{T \bar{w}} \sum_{t=1}^{T} w\left(X_{t}\right) I\left(\varepsilon_{t} \leq y\right)\left(\varepsilon_{t}-\widehat{\varepsilon}_{t}\right) \\
& \quad+\frac{1}{T \bar{w}} \sum_{t=1}^{T} w\left(X_{t}\right)\left\{I\left(\widehat{\varepsilon}_{t} \leq y\right)-I\left(\varepsilon_{t} \leq y\right)\right\}\left(y-\widehat{\varepsilon}_{t}\right) \\
& =A(y)+B(y) \quad \text { (say). }
\end{aligned}
$$


When $\widehat{\varepsilon}_{t}$ is replaced by $\widehat{\varepsilon}_{t 0}$ in the above sum, we get a similar decomposition which we denote by $A_{0}(y)+B_{0}(y)$. First, consider

$$
\begin{aligned}
A(y) & -A_{0}(y) \\
& =\frac{1}{T \bar{w}} \sum_{t=1}^{T} w\left(X_{t}\right) I\left(\varepsilon_{t} \leq y\right)\left(\widehat{\varepsilon}_{t 0}-\widehat{\varepsilon}_{t}\right) \\
& =\frac{1}{T \bar{w}} \sum_{t=1}^{T} w\left(X_{t}\right) I\left(\varepsilon_{t} \leq y\right) \frac{\widehat{\mu}\left(X_{t}\right)-\widehat{\mu}_{0}\left(X_{t}\right)}{\widehat{\sigma}\left(X_{t}\right)} \\
& =\frac{1}{T E(w(X))} \sum_{t=1}^{T} w\left(X_{t}\right) I\left(\varepsilon_{t} \leq y\right) \frac{\widehat{\mu}\left(X_{t}\right)-\widehat{\mu}_{0}\left(X_{t}\right)}{\sigma\left(X_{t}\right)}+o_{P}\left(T^{-1 / 2}\right) .
\end{aligned}
$$

We will now show that

$$
\begin{aligned}
& \frac{1}{T E(w(X))} \sum_{t=1}^{T}\left[w\left(X_{t}\right) I\left(\varepsilon_{t} \leq y\right) \frac{\widehat{\mu}\left(X_{t}\right)-\widehat{\mu}_{0}\left(X_{t}\right)}{\sigma\left(X_{t}\right)}\right. \\
& \left.\quad-E\left\{w(X) I(\varepsilon \leq y) \frac{\widehat{\mu}(X)-\widehat{\mu}_{0}(X)}{\sigma(X)}\right\}\right]=o_{P}\left(T^{-1 / 2}\right),
\end{aligned}
$$

uniformly in $-\infty<y<\infty$, where the latter expectation is taken conditional on $\widehat{\mu}$ and $\widehat{\mu}_{0}$, and where the pair $(X, \varepsilon)$ has the same distribution as $\left(X_{t}, \varepsilon_{t}\right)$ and is independent of $\left(X_{1}, \varepsilon_{1}\right), \ldots,\left(X_{T}, \varepsilon_{T}\right)$. Define the class

$$
\mathcal{F}=\left\{(x, e) \rightarrow w(x) \sigma^{-1}(x) I(e \leq y) v(x):-\infty<y<+\infty, v \in C_{M}^{\tau}\left(R_{w}\right)\right\},
$$

where $C_{M}^{\tau}\left(R_{w}\right)$ is the space of continuous functions $v$ defined on the compact set $R_{w}$, for which

$$
\|v\|_{\tau}=\max _{k . \leq \underline{\tau}} \sup _{x}\left|D^{k} v(x)\right|+\max _{k .=\underline{\tau} x, x^{\prime}} \frac{\left|D^{k} v(x)-D^{k} v\left(x^{\prime}\right)\right|}{\left\|x-x^{\prime}\right\|^{\tau-\underline{\tau}}} \leq M<\infty,
$$

where $\tau$ is the largest integer strictly smaller than $\tau, k=\left(k_{1}, \ldots, k_{d}\right), D^{k}=$ $\partial^{k} \cdot \partial x_{1}^{k_{1}} \cdots \partial x_{d}^{k_{d}}$, and $k .=\sum k_{i}$. Note that $P\left(\widehat{\mu}-\widehat{\mu}_{0} \in C_{M}^{\tau}\left(R_{w}\right)\right) \rightarrow 1$ as $T$ tends to infinity, by Assumptions G3(ii) and G4(ii). We will show that this class is Donsker. A sufficient condition for the class $\mathcal{F}$ to be Donsker is that

$$
\int_{0}^{2 M} \sqrt{\log N_{[\cdot]}\left(\delta, \mathcal{F},\|\cdot\|_{2, \beta}\right)} d \delta<\infty,
$$

where for any function $g,\|g\|_{2, \beta}^{2}=\int_{0}^{1} \beta^{-1}(u) Q_{g}^{2}(u) d u$, and where $\beta^{-1}$ is the inverse cadlag of the decreasing function $u \rightarrow \beta_{\lfloor u\rfloor}(\lfloor u\rfloor$ being the integer part of $u$, and $\beta_{t}$ being the mixing coefficient) and $Q_{g}$ is the inverse cadlag of the tail function $u \rightarrow P\left(\|g\|>u\right.$ ) (see Section 4.3 in [3]). Here, $N_{[]}\left(\delta, \mathcal{F},\|\cdot\|_{2, \beta}\right)$ is the $\delta$-bracketing number of the class $\mathcal{F}$, that is, it is the smallest number of $\delta$-brackets 
needed to cover the space $\mathcal{F}$, where a $\delta$-bracket is the set of all functions $h$ such that $h_{\ell} \leq h \leq h_{u}$ and where $\left(h_{\ell}, h_{u}\right)$ satisfy $\left\|h_{u}-h_{\ell}\right\|_{2, \beta} \leq \delta$. From Corollary 2.7.2 in [37], it follows that $N_{[]}\left(\delta, C_{M}^{\tau}\left(R_{w}\right),\|\cdot\|_{2}\right) \leq \exp \left(K \delta^{-d / \tau}\right)$. Moreover, defining $y_{j}=F_{\varepsilon}^{-1}(j \delta)$ for $j=1, \ldots, O\left(\delta^{-1}\right)$, it is easily seen that $N_{[]}\left(\delta, \mathcal{F},\|\cdot\|_{2, \beta}\right)=$ $O\left(\delta^{-2} \exp \left(K \delta^{-d / \tau}\right)\right.$ ) (see, e.g., the proof of Lemma 1 in [7]). It now follows that the class $\mathcal{F}$ is Donsker, provided $\tau>d / 2$. Next, note that

$$
\operatorname{Var}\left\{w(X) I(\varepsilon \leq y) \frac{\widehat{\mu}(X)-\widehat{\mu}_{0}(X)}{\sigma(X)}\right\} \leq K \sup _{x \in R_{w}}\left|\widehat{\mu}(x)-\widehat{\mu}_{0}(x)\right|^{2} \stackrel{P}{\rightarrow} 0,
$$

where the variance is conditional on $\widehat{\mu}$ and $\widehat{\mu}_{0}$, and where the pair $(X, \varepsilon)$ is as before independent of $\widehat{\mu}$ and $\widehat{\mu}_{0}$. Hence, it follows from Corollary 2.3.12 in [37] that (14) holds true. It remains to calculate

$$
\begin{aligned}
& \frac{1}{E(w(X))} E\left\{w(X) I(\varepsilon \leq y) \frac{\widehat{\mu}(X)-\widehat{\mu}_{0}(X)}{\sigma(X)}\right\} \\
& =-\frac{F_{\varepsilon}(y)}{E(w(X))} \int w(x) \frac{\widehat{\mu}_{0}(x)-\mu_{0}(x)}{\sigma(x)} d F_{X}(x) \\
& \quad+\frac{F_{\varepsilon}(y)}{E(w(X))} \int w(x) \frac{\widehat{\mu}(x)-\mu(x)}{\sigma(x)} d F_{X}(x),
\end{aligned}
$$

which follows from the independence between $X$ and $\varepsilon$.

By Assumption G3(iii),

$$
\int \frac{w(x)}{\sigma(x)}(\widehat{\mu}(x)-\mu(x)) d F_{X}(x)=T^{-1} \sum_{t=1}^{T} w\left(X_{t}\right) l\left(Y_{t}, X_{t}\right)+o_{P}\left(T^{-1 / 2}\right),
$$

whereas by Assumption G4(iii) under the null hypothesis

$$
\int \frac{w(x)}{\sigma(x)}\left(\widehat{\mu}_{0}(x)-\mu(x)\right) d F_{X}(x)=T^{-1} \sum_{t=1}^{T} w\left(X_{t}\right) l_{0}\left(Y_{t}, X_{t}\right)+o_{P}\left(T^{-1 / 2}\right) .
$$

Next, consider the term $B(y)$, which can be bounded as follows:

$$
\begin{aligned}
B(y) & =\frac{1}{T \bar{w}} \sum_{t=1}^{T} w\left(X_{t}\right)\left[I\left(\widehat{\varepsilon}_{t} \leq y<\varepsilon_{t}\right)\left(y-\widehat{\varepsilon}_{t}\right)-I\left(\varepsilon_{t} \leq y<\widehat{\varepsilon}_{t}\right)\left(y-\widehat{\varepsilon}_{t}\right)\right] \\
& \leq \frac{1}{T \bar{w}} \sum_{t=1}^{T} w\left(X_{t}\right)\left[I\left(\widehat{\varepsilon}_{t} \leq y<\varepsilon_{t}\right)\left(\varepsilon_{t}-\widehat{\varepsilon}_{t}\right)+I\left(\varepsilon_{t} \leq y<\widehat{\varepsilon}_{t}\right)\left(\widehat{\varepsilon}_{t}-\varepsilon_{t}\right)\right] .
\end{aligned}
$$

Each of the above terms is $o_{P}\left(T^{-1 / 2}\right)$ uniformly in $y$. Indeed, the first term on the right-hand side is bounded by

$$
\frac{1}{T \bar{w}} \max _{t: w\left(X_{t}\right)>0}\left|\widehat{\varepsilon}_{t}-\varepsilon_{t}\right| \sum_{t=1}^{T} w\left(X_{t}\right) I\left(\widehat{\varepsilon}_{t} \leq y<\varepsilon_{t}\right) .
$$


Note that [where the probability $P(\widehat{\varepsilon} \leq y)$ is conditional on $\widehat{\mu}$ and $\widehat{\sigma}$, and where the pair $(X, \varepsilon)$ is as before independent of $\widehat{\mu}$ and $\widehat{\sigma}]$

$$
\begin{aligned}
\frac{1}{T} \sum_{t=1}^{T} w\left(X_{t}\right) I\left(\widehat{\varepsilon}_{t} \leq y<\varepsilon_{t}\right) \\
=\frac{1}{T} \sum_{t=1}^{T}\left[w\left(X_{t}\right)\left(I\left(\widehat{\varepsilon}_{t} \leq y\right)-I\left(\varepsilon_{t} \leq y\right)\right)-E\{w(X)(I(\widehat{\varepsilon} \leq y)-I(\varepsilon \leq y))\}\right] \\
\quad+E[w(X)(I(\widehat{\varepsilon} \leq y)-I(\varepsilon \leq y))] \\
\leq \sup _{x \in R_{w}} w(x)\left\{\sup _{y} f_{\varepsilon}(y) \sup _{x \in R_{w}}|\widehat{\mu}(x)-\mu(x)|\right. \\
\left.\quad+\sup _{y}\left|y f_{\varepsilon}(y)\right| \sup _{x \in R_{w}}|\widehat{\sigma}(x)-\sigma(x)|\right\}+o_{P}\left(T^{-1 / 2}\right),
\end{aligned}
$$

uniformly in $y$ (where the term $o_{P}\left(T^{-1 / 2}\right)$ in the last line follows from a combination of the above arguments for the term $A(y)$ and the proof of Lemma 1 in [7]). Hence, the first term of $B(y)$ is of the order $O_{P}\left(\sup _{x \in R_{w}}|\widehat{\mu}(x)-\mu(x)|^{2}\right)+$ $O_{P}\left(\sup _{x \in R_{w}}|\widehat{\sigma}(x)-\sigma(x)|^{2}\right)+o_{P}\left(T^{-1 / 2}\right)=o_{P}\left(T^{-1 / 2}\right)$ by Assumption G3(i). The second term of $B(y)$ can be bounded in a similar way. Finally, it can be shown that $B_{0}(y)$ is also $o_{P}\left(T^{-1 / 2}\right)$ by using Assumption G4(i).

Therefore, by the arguments above, uniformly in $y$ :

$$
\begin{aligned}
\int_{-\infty}^{y} & {\left[\widehat{F}_{\varepsilon 0}(s)-\widehat{F}_{\varepsilon}(s)\right] d s } \\
& =A_{0}(y)-A(y)+o_{P}\left(T^{-1 / 2}\right) \\
& =\frac{F_{\varepsilon}(y)}{T E\left(w\left(X_{t}\right)\right)} \sum_{t=1}^{T} w\left(X_{t}\right) W_{t}+o_{P}\left(T^{-1 / 2}\right) .
\end{aligned}
$$

This completes the proof.

\section{SUPPLEMENTARY MATERIAL}

Supplement to "Asymptotic distribution-free tests for semiparametric regressions with dependent data" (DOI: 10.1214/17-AOS1581SUPP; .pdf). The supplement contains further Monte Carlo simulations and the proofs of some asymptotic results.

\section{REFERENCES}

[1] BekAert, G. and Harvey, C. R. (1995). Time-varying world market integration. J. Finance 50 403-444.

[2] CHEN, X. (2007). Large sample sieve estimation of semi-nonparametric models. In Handbook of Econometrics (J. J. Heckman and E. E. Leamer, eds.) 6 5549-5632. 
[3] Dedecker, J. and Louhichi, S. (2002). Maximal inequalities and empirical central limit theorems. In Empirical Process Techniques for Dependent Data (H. Dehling, T. Mikosch and M. Sørensen, eds.) 137-159. Birkhäuser, Boston, MA. MR1958779

[4] Delgado, M. A. and GonZÁlez Manteiga, W. (2001). Significance testing in nonparametric regression based on the bootstrap. Ann. Statist. 29 1469-1507. MR1873339

[5] Dette, H., Marchlewski, M. and Wagener, J. (2012). Testing for a constant coefficient of variation in nonparametric regression by empirical processes. Ann. Inst. Statist. Math. 64 1045-1070. MR2960957

[6] Dette, H., Neumeyer, N. and Van Keilegom, I. (2007). A new test for the parametric form of the variance function in non-parametric regression. J. R. Stat. Soc. Ser. B. Stat. Methodol. 69 903-917. MR2368576

[7] Dette, H., Pardo-Fernández, J. C. and Van Keilegom, I. (2009). Goodness-of-fit tests for multiplicative models with dependent data. Scand. J. Stat. 36 782-799. MR2573308

[8] De SANTIS, G. and Gerard, B. (1997). International asset pricing and portfolio diversification with time-varying risk. J. Finance 52 1881-1912.

[9] Doukhan, P. (1994). Mixing. Lecture Notes in Statistics: Properties and Examples 85. Springer, New York. MR1312160

[10] Einmahl, U. and MAson, D. M. (2005). Uniform in bandwidth consistency of kernel-type function estimators. Ann. Statist. 33 1380-1403. MR2195639

[11] EsCANCIANO, J. C. (2009). On the lack of power of omnibus specification tests. Econometric Theory 25 162-194. MR2472049

[12] Escanciano, J. C., Jacho-Chávez, D. T. and Lewbel, A. (2014). Uniform convergence of weighted sums of non and semiparametric residuals for estimation and testing. J. Econometrics 178 426-443. MR3132442

[13] Escanciano, J. C., Pardo-Fernández, J. C. and Van Keilegom, I. (2017). Semiparametric estimation of risk-return relationships. J. Bus. Econom. Statist. 35 40-52. MR3591536

[14] Escanciano, J. C., Pardo-Fernández, J. C. and Van Keilegom, I. (2018). Supplement to "Asymptotic distribution-free tests for semiparametric regressions with dependent data." DOI:10.1214/17-AOS1581SUPP.

[15] FAN, J. and YAO, Q. (1998). Efficient estimation of conditional variance functions in stochastic regression. Biometrika 85 645-660. MR1665822

[16] FAn, J. and YAO, Q. (2003). Nonlinear Time Series: Nonparametric and Parametric Methods. Springer, New York. MR1964455

[17] Ferson, W. (1989). Changes in expected security returns, risk and level of interest rates. J. Finance 44 1191-1217.

[18] Ferson, W., Foerster, S. R. and Keim, D. B. (1993). General tests of latent variable models and mean-variance spanning. J. Finance 48 131-156.

[19] GonZÁlez-Manteiga, W. and Crujeiras, R. M. (2013). An updated review of goodnessof-fit tests for regression models. TEST 22 361-411. MR3093195

[20] Hansen, B. E. (2008). Uniform convergence rates for kernel estimation with dependent data. Econometric Theory 24 726-748. MR2409261

[21] Härdle, W., Hall, P. and Ichimura, H. (1993). Optimal smoothing in single-index models. Ann. Statist. 21 157-178. MR1212171

[22] HÄrdLE, W. and MAMMEN, E. (1993). Comparing nonparametric versus parametric regression fits. Ann. Statist. 21 1926-1947. MR1245774

[23] Harvey, C. R. (1989). Time-varying conditional covariances in tests of asset pricing models. J. Financ. Econom. 24 289-317.

[24] Janssen, A. (2000). Global power functions of goodness of fit tests. Ann. Statist. 28 239-253. MR1762910 
[25] Khmaladze, E. V. and Koul, H. L. (2004). Martingale transforms goodness-of-fit tests in regression models. Ann. Statist. 32 995-1034. MR2065196

[26] Koul, H. L. and STUTE, W. (1999). Nonparametric model checks for time series. Ann. Statist. 27 204-236. MR1701108

[27] LeE, T.-H., TU, Y. and Ullah, A. (2015). Forecasting equity premium: Global historical average versus local historical average and constraints. J. Bus. Econom. Statist. $33393-$ 402. MR3372666

[28] MASRY, E. (1996). Multivariate local polynomial regression for time series: Uniform strong consistency and rates. J. Time Series Anal. 17 571-599. MR1424907

[29] Merton, R. C. (1973). An intertemporal capital asset pricing model. Econometrica 41 867887. MR0441271

[30] Merton, R. C. (1980). On estimating the expected return on the market. An explanatory investigation. J. Financ. Econom. 8 323-361.

[31] Neumeyer, N. and VAN Keilegom, I. (2010). Estimating the error distribution in nonparametric multiple regression with applications to model testing. J. Multivariate Anal. 101 1067-1078. MR2595293

[32] NewEy, W. K. (1994). The asymptotic variance of semiparametric estimators. Econometrica 62 1349-1382. MR1303237

[33] Robinson, P. M. (1988). Root- $N$-consistent semiparametric regression. Econometrica 56 931-954. MR0951762

[34] Stute, W. (1997). Nonparametric model checks for regression. Ann. Statist. 25 613-641. MR1439316

[35] Stute, W., XU, W. L. and ZHU, L. X. (2008). Model diagnosis for parametric regression in high-dimensional spaces. Biometrika 95 451-467. MR2521592

[36] TANG, Y. and WhitelaW, R. F. (2011). Time-varying sharpe ratios and market timing. Quarterly J. Finance 1 465-493.

[37] VAN DER VAART, A. W. and Wellner, J. A. (1996). Weak Convergence and Empirical Processes: With Applications to Statistics. Springer, New York. MR1385671

[38] Van Keilegom, I., González Manteiga, W. and Sánchez Sellero, C. (2008). Goodness-of-fit tests in parametric regression based on the estimation of the error distribution. TEST 17 401-415. MR2434335

\section{J. C. ESCANCIANO \\ DEPARTMENT OF ECONOMICS \\ INDIANA UNIVERSITY \\ Wylie Hall, $100 \mathrm{~S}$ WoOdLaWn AVE \\ BLOOMINGTON, INDIANA 47405 \\ USA \\ E-MAIL: jescanci@indiana.edu}

\author{
J. C. PARDO-FERNÁNDEZ \\ DEPARTAMENTO DE ESTATÍSTICA E IO \\ UNIVERSIDADE DE VIGO \\ Campus Universitario As Lagoas-Marcosende \\ 36310 VIGO \\ SPAIN \\ E-MAIL: juancp@uvigo.es
}

\author{
I. VAN KEILEGOM \\ ORSTAT \\ KU LEUVEN \\ NAAMSESTRAAT 69 \\ 3000 LEUVEN \\ BELGIUM \\ E-MAIL: ingrid.vankeilegom@kuleuven.be
}

